Supporting Information for IC 0610381

Active Site Models for Fe Hydrogenases: Reduction Chemistry of Dinuclear Iron

\title{
Complexes
}

Inigo Aguirre de Carcer, Antonio DiPasquale, Arnold L. Rheingold and

D. Michael Heinekey*

Department of Chemistry, University of Washington, Box 351700

Seattle, WA 98195-1700

Department of Chemistry and Biochemistry, University of California San Diego, La

Jolla, CA 92093-0332

I. Synthesis of 2 .

II. Quantitative I.R. spectroscopy of the reduction of 1 and reoxidation of 2. S3

III. Quantitative I.R. spectroscopy of the reduction of 3 and reoxidation of 4. S4

III. ${ }^{1} \mathrm{H}-\mathrm{NMR}$ of complex $4 . \quad$ S5

IV. Oxidation of 2 and 4 with $\mathrm{H}^{+}$. S6

$\begin{array}{ll}\text { V. X ray-Crystallographic data of } 2 \text { S7 } & \text { S7 }\end{array}$ 
Experimental. THF and $\mathrm{Et}_{2} \mathrm{O}$ were dried over $\mathrm{Na} / \mathrm{K}$ amalgam in the presence of benzophenone. $\mathrm{Cp}_{2}{ }_{2} \mathrm{Co}$ was purchased from Aldrich and sublimed $\left(100^{\circ} \mathrm{C}, 4\right.$ torr $)$ prior to use. $\mathrm{Fe}_{2}$ (u-pdt) $\mathrm{CO}_{6}$ was prepared by literature methods. (E. J. Lyon; I. P. Georgakaki; J. H. Reibenspies; M. Y. Darensbourg. J. Am. Chem. Soc., 123, 2001. 3268). The aza bridged species $\mathrm{Fe}_{2}\left(\mathrm{SCH}_{2} \mathrm{NPhCH}_{2} \mathrm{~S}\right)(\mathrm{CO})_{6}$ was prepared by a modification of the reported method. (S. Ott; M. Kritikos; B. Åkermark; L. Sun. Angew. Chem. Intl. Ed. Engl., 2003. 42. 3285). $\left[\mathrm{Cp}_{2} \mathrm{Fe}\right]\left[\mathrm{PF}_{6}\right]$ was purchased from Aldrich and recrystallized from acetone/ethanol. All IR spectra were recorded with a Bruker Optics Tensor 27 instrument. ${ }^{1} \mathrm{H}-\mathrm{NMR}$ spectra were obtained using a Bruker DRX spectrometer operating at $499 \mathrm{MHz}$.

\section{Synthesis of $\left[\mathrm{Fe}_{2}\left(\mu-\mathrm{S}_{2} \mathrm{C}_{3} \mathrm{H}_{6}\right) \mathrm{CO}_{5}\left(\mathrm{SCH}_{2} \mathrm{CH}_{2} \mathrm{CH}_{2}-\mu-\mathrm{S}\right)-\mathrm{Fe}_{2}(\mu-\mathrm{CO})(\mathrm{CO})_{6}\right]\left[\mathrm{Cp}_{2}{ }_{2} \mathrm{Co}_{2}(2)\right.$.}

$60 \mathrm{mg}(15.5 \mathrm{mmol})$ of $\mathrm{Fe}_{2}(\mathrm{u}-\mathrm{pdt}) \mathrm{CO}_{6}(\mathbf{1})$ and $52 \mathrm{mg}(15.8 \mathrm{mmol})$ of freshly sublimed $\mathrm{Cp}^{*}{ }_{2} \mathrm{Co}$ were placed in an H-tube. THF $(4 \mathrm{~mL})$ was vacuum transferred into the reaction vessel. The solution was stirred briefly, then $\mathrm{Et}_{2} \mathrm{O}$ was added by vacuum transfer with stirring until a precipitate appears. After the brown precipitate settles the supernatant is decanted to the empty side of the H-tube and then pumped away. The solid is washed with two $8 \mathrm{~mL}$ portions of $\mathrm{Et}_{2} \mathrm{O}$. After pumping overnight, $90 \mathrm{mg}(6.3 \mathrm{mmol})$ of 2 was recovered as an orange powder, 81\% yield. IR(KBr): 2018, 1967, 1940, 1930, 1915,

$1883 \mathrm{~cm}^{-1}$ and $1730 \mathrm{~cm}^{-1}$. Elemental analysis: $\mathrm{C}_{58} \mathrm{H}_{72} \mathrm{Co}_{2} \mathrm{Fe}_{4} \mathrm{O}_{12} \mathrm{~S}_{4}$ Calculated:C 48.68, $\mathrm{H}$ 5.08, S 8.96. Found: C 48.50, H 5.30, S 8.99. 


\section{Quantitative I.R. spectroscopy of the reduction of 1 and reoxidation of 2.}

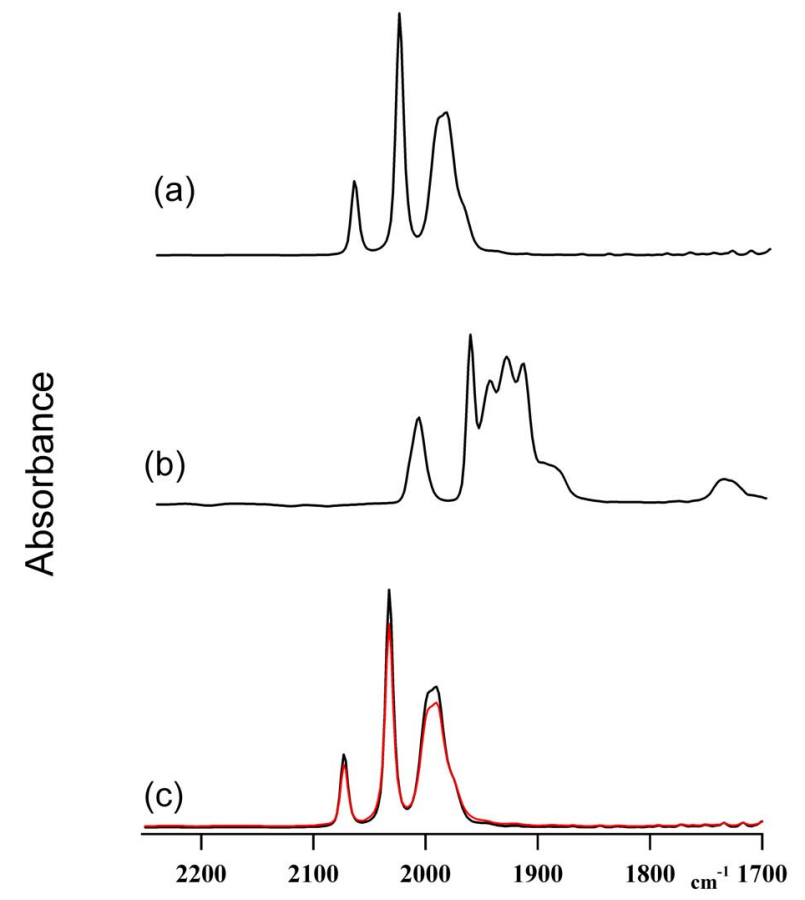

Partial infrared spectra(CO stretching region, THF solutions)

(a) Complex 1 ( 0.020g, 0.051 mmol in $20 \mathrm{~mL}$ of THF) 2072, 2033, $1993 \mathrm{~cm}^{-1}$.

(b) complete formation of 2 after treatment of (a) with $\mathrm{Cp}_{2}{ }_{2} \mathrm{Co}(0.0173 \mathrm{~g}, 0.053 \mathrm{mmol})$. (c) oxidation of (b) with solid $\left[\mathrm{Cp}_{2} \mathrm{Fe}\right]\left[\mathrm{PF}_{6}\right](0.0175 \mathrm{~g}, 0.053 \mathrm{mmol})$ in red, compared with 1 before reaction in black.

Integration of the spectra before and after reaction with Bruker OPUS software provides a yield for the overall reaction of $90 \%$. 


\section{Reduction of 3 and reoxidation of 4 .}

(a)
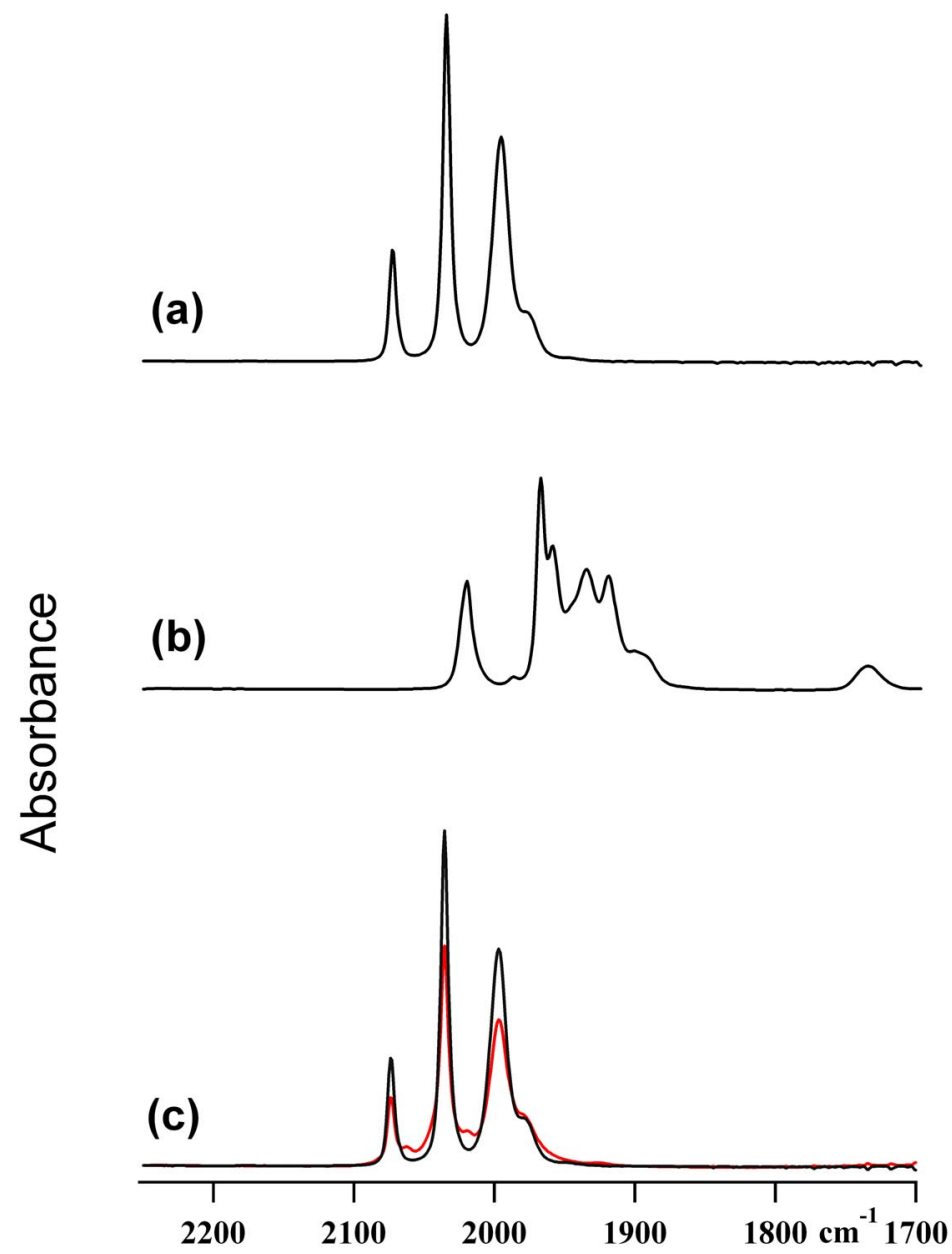

Partial infrared spectra(CO stretching region, THF solutions)

(a) Complex 3 ( 0.012g, 0.026mmol in $10 \mathrm{~mL}$ of THF) 2074, 2036, $1997 \mathrm{~cm}^{-1}$.

(b) complete formation of 4 after treatment of (a) with of solid $\mathrm{Cp}_{2}{ }_{2} \mathrm{Co}$ ( $0.0094 \mathrm{~g}$. $0.028 \mathrm{mmol}$ )Bands due to 4 are observed at 2021, 1969, 1960, 1936, 1920, $1738 \mathrm{~cm}^{-1}$. (c) in red oxidation of (b) with solid $\left[\mathrm{Cp}_{2} \mathrm{Fe}\right]\left[\mathrm{PF}_{6}\right](0.098 \mathrm{~g}, 0.029 \mathrm{mmol})$ compared with 3 before reaction in black.

Integration of the spectra before and after reaction with Bruker OPUS software provides a yield for the overall reaction of $70 \%$. 
IV. ${ }^{1}$ H NMR spectrum of complex 4 .

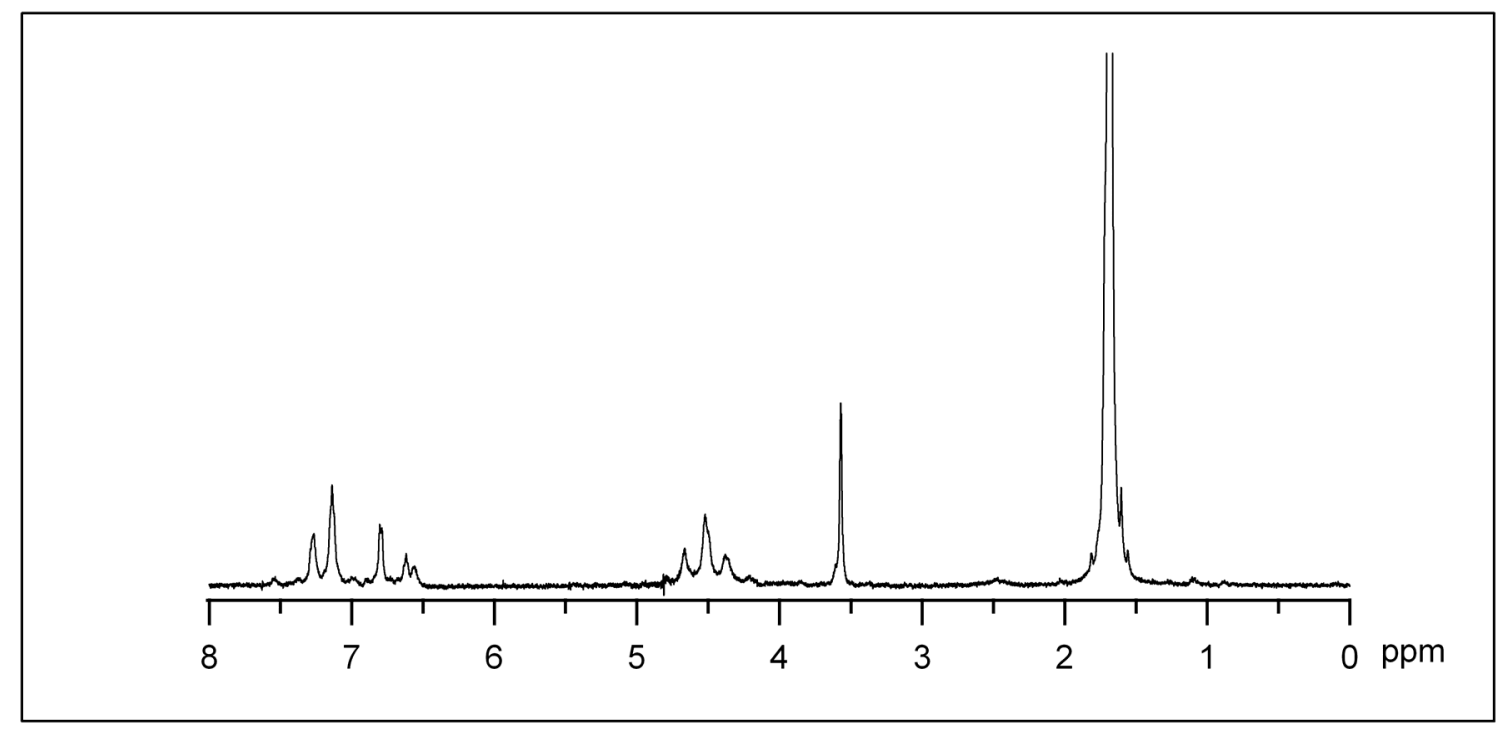

$500 \mathrm{MHz}{ }^{1} \mathrm{H}-\mathrm{NMR}$ spectrum of complex 4 in THF- $\mathrm{d}_{8}$. Chemical shifts in ppm are: 7.26(2H), 7.14(4H), 6.8(2H), 6.62(1H), 6.55(1H), 4.63(2H), 4.50(4H), 4.38(2H), 1.70(60H). 


\section{Oxidation of 2 and 4 with acids.}
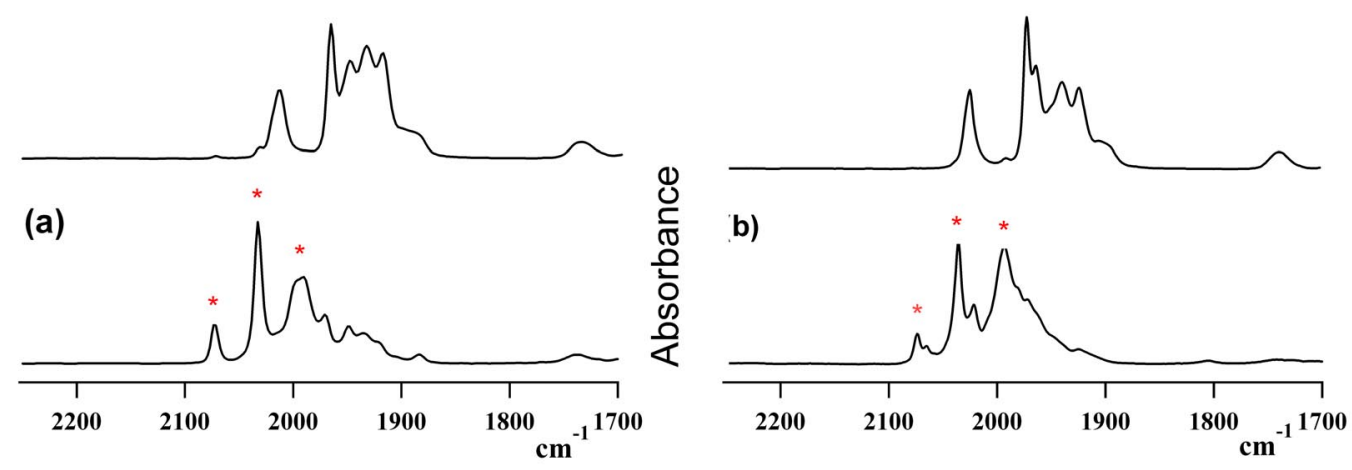

Partial IR spectra (CO stretching region, THF solutions).

(a) Top: complex 2. Bottom: Complex 2 after reaction with 2.2 equivalents of ptoluenesulphonic acid (red asterisk identifies bands due to $\mathrm{Fe}_{2}\left(\mu-\mathrm{S}_{2} \mathrm{C}_{3} \mathrm{H}_{6}\right) \mathrm{CO}_{6}$, (1). The same experiment carried out in an NMR tube shows after reaction the resonances at 2.2 ppm $(4 \mathrm{H})$ and 1.9 ppm $(2 \mathrm{H})$ associated with $\mathrm{Fe}_{2}\left(\mu-\mathrm{S}_{2} \mathrm{C}_{3} \mathrm{H}_{6}\right) \mathrm{CO}_{6}$ and a resonance at 4.6 ppm attributed to dissolved $\mathrm{H}_{2}$ gas. (b) Top: Complex 4. Bottom: 4 after reaction with 2.2 equivalents of p-toluenesulphonic acid (red asterisk indicates bands associated with $\left.\mathrm{Fe}_{2}\left(\mathrm{SCH}_{2} \mathrm{NPhCH}_{2} \mathrm{~S}\right)(\mathrm{CO})_{6}\right)$. 


\section{X ray-Crystallographic Analysis for Complex 2 .}

A brown plate $0.20 \times 0.20 \times 0.05 \mathrm{~mm}$ in size was mounted on a cryoloop with Paratone ${ }^{\circledR}$ oil. Data was collected in a nitrogen gas stream at $-173^{\circ} \mathrm{C}$. Crystal-todetector distance was $60 \mathrm{~mm}$ and exposure time was 20 seconds per frame using a scan width of $0.3^{\circ}$. Data collection was $99.5 \%$ complete to $25^{\circ}$ in $\theta$. A total of 31,845 reflections were collected covering the indices, $\mathrm{h}=-10$ to $10, \mathrm{k}=-22$ to $22,1=-23$ to 23 . 10,671 reflections were found to be symmetry independent, with an $R_{\text {int }}$ of 0.1038 indicating that the data was of less than average quality (0.07). Indexing and unit cell refinement indicated a $P$ centered, triclinic lattice. The space group was found to be $P-1$ (No. 2). The data was integrated using the Bruker SAINT software program and scaled using the Bruker SADABS software program. Solution by direct methods (SIR-97) produced a complete heavy atom phasing model consistent with the proposed structure. All non-hydrogen atoms were refined anisotropically by full-matrix least-squares (SHELXL-97). All hydrogen atoms were placed using a riding model and their positions constrained relative to their parent atom using the appropriate HFIX command in SHELXL-97. 


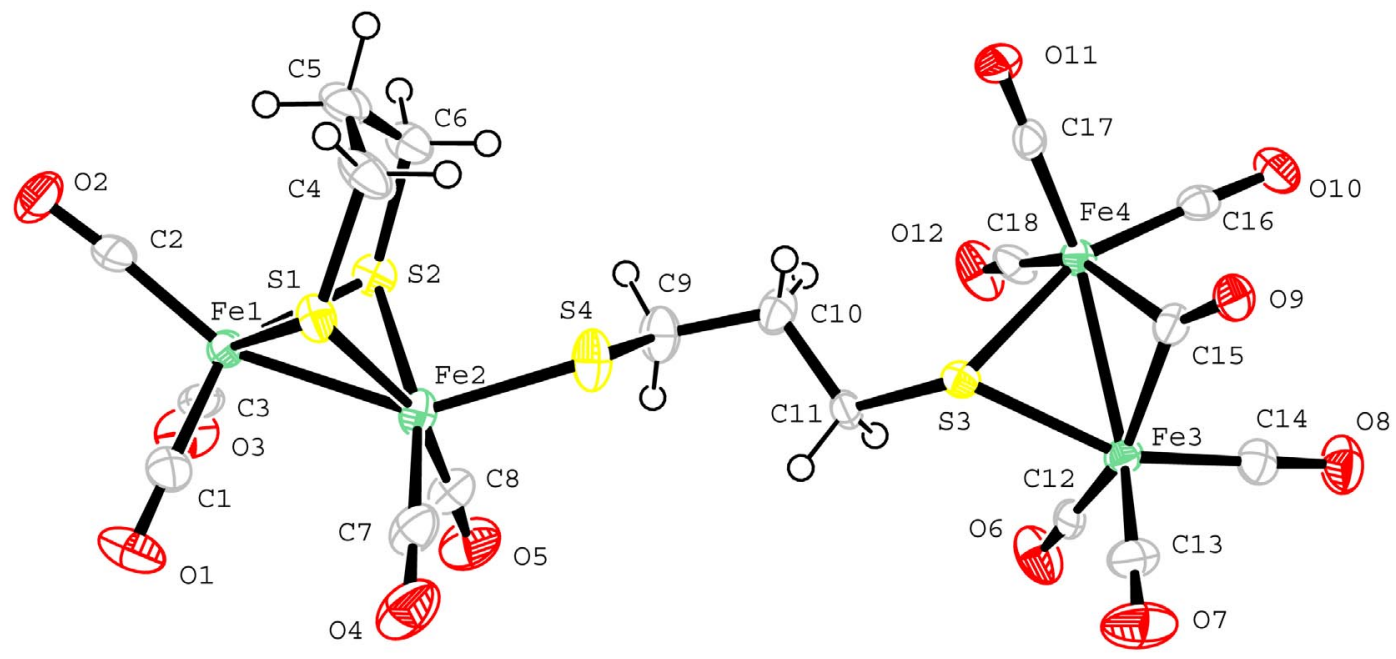

Table 1. Crystal data and structure refinement for 2.

Empirical formula

Formula weight

Temperature

Wavelength

Crystal system

Space group

Unit cell dimensions

Volume

$\mathrm{Z}$

Density (calculated)

Absorption coefficient

$\mathrm{F}(000)$

Crystal size

Theta range for data collection

Index ranges

Reflections collected
C58 H72 Co2 Fe4 O12 S4

1430.66

100(2) K

$0.71069 \AA$

Triclinic

P-1

$\mathrm{a}=9.163(6) \AA$ $\square=116.615(9)^{\circ}$.

$\mathrm{b}=19.047(13) \AA$ $\square=97.989(11)^{\circ}$.

$\mathrm{c}=20.123(14) \AA$ $\square=95.037(10)^{\circ}$.

3064(4) $\AA^{3}$

2

$1.550 \mathrm{Mg} / \mathrm{m}^{3}$

$1.645 \mathrm{~mm}^{-1}$

1476

$0.20 \times 0.20 \times 0.05 \mathrm{~mm}^{3}$

2.05 to $25.02^{\circ}$.

$-10<=\mathrm{h}<=10,-22<=\mathrm{k}<=22,-23<=1<=23$

31845 
Independent reflections

Completeness to theta $=25.00^{\circ}$

Absorption correction

Max. and min. transmission

Refinement method

Data / restraints / parameters

Goodness-of-fit on $\mathrm{F}^{2}$

Final $\mathrm{R}$ indices [I $>2 \operatorname{sigma}(\mathrm{I})]$

$\mathrm{R}$ indices (all data)

Largest diff. peak and hole
$10761[\mathrm{R}($ int $)=0.1038]$

$99.5 \%$

Semi-empirical from equivalents

0.9223 and 0.7344

Full-matrix least-squares on $\mathrm{F}^{2}$

$10761 / 0 / 744$

0.924

$\mathrm{R} 1=0.0467, \mathrm{wR} 2=0.0890$

$\mathrm{R} 1=0.0729, \mathrm{wR} 2=0.0960$

1.069 and -0.673 e. $\AA^{-3}$

Table 2. Atomic coordinates ( $\left.\times 10^{4}\right)$ and equivalent isotropic displacement parameters $\left(\AA^{2} \times 10^{3}\right)$ for 2. $U(\mathrm{eq})$ is defined as one third of the trace of the orthogonalized $\mathrm{U}^{\mathrm{ij}}$ tensor.

\begin{tabular}{lcccc}
\hline & $\mathrm{x}$ & $\mathrm{y}$ & $\mathrm{z}$ & $\mathrm{U}(\mathrm{eq})$ \\
\hline $\mathrm{C}(1)$ & $2125(6)$ & $4219(2)$ & $3805(3)$ & $30(1)$ \\
$\mathrm{C}(2)$ & $-527(5)$ & $4842(3)$ & $3980(3)$ & $32(1)$ \\
$\mathrm{C}(3)$ & $-278(5)$ & $3297(3)$ & $3496(2)$ & $32(1)$ \\
$\mathrm{C}(4)$ & $-98(5)$ & $5082(3)$ & $2258(3)$ & $36(1)$ \\
$\mathrm{C}(5)$ & $-1705(6)$ & $4859(3)$ & $2286(3)$ & $37(1)$ \\
$\mathrm{C}(6)$ & $-2276(5)$ & $3977(2)$ & $1836(2)$ & $32(1)$ \\
$\mathrm{C}(7)$ & $3014(6)$ & $3480(3)$ & $2219(3)$ & $36(1)$ \\
$\mathrm{C}(8)$ & $907(5)$ & $2361(3)$ & $1834(3)$ & $34(1)$ \\
$\mathrm{C}(9)$ & $-655(5)$ & $2324(3)$ & $247(2)$ & $34(1)$ \\
$\mathrm{C}(10)$ & $-766(5)$ & $2137(3)$ & $-575(2)$ & $31(1)$
\end{tabular}




\begin{tabular}{|c|c|c|c|c|}
\hline $\mathrm{C}(11)$ & $470(5)$ & $1725(2)$ & $-937(2)$ & $23(1)$ \\
\hline$C(12)$ & $1072(5)$ & 293(2) & $-2464(2)$ & $25(1)$ \\
\hline $\mathrm{C}(13)$ & $3422(6)$ & 1379(3) & $-2523(3)$ & $32(1)$ \\
\hline$C(14)$ & $1131(5)$ & 391(3) & $-3693(3)$ & $27(1)$ \\
\hline$C(15)$ & $829(5)$ & $1869(2)$ & $-3020(2)$ & $24(1)$ \\
\hline$C(16)$ & $-1910(5)$ & $1224(2)$ & $-3581(3)$ & $23(1)$ \\
\hline $\mathrm{C}(17)$ & $-1482(5)$ & 2624(3) & $-2302(2)$ & $23(1)$ \\
\hline$C(18)$ & $-2080(5)$ & 1045(3) & $-2384(2)$ & $26(1)$ \\
\hline$C(19)$ & $2931(5)$ & $2848(2)$ & $-5112(2)$ & $22(1)$ \\
\hline $\mathrm{C}(20)$ & $3020(5)$ & 2299(3) & $-5871(2)$ & $29(1)$ \\
\hline$C(21)$ & $4354(6)$ & 2569(3) & $-6033(2)$ & $32(1)$ \\
\hline $\mathrm{C}(22)$ & $5103(5)$ & $3275(3)$ & $-5378(2)$ & $26(1)$ \\
\hline$C(23)$ & $4243(5)$ & $3454(2)$ & $-4813(2)$ & $20(1)$ \\
\hline $\mathrm{C}(24)$ & $1700(5)$ & 2814(3) & $-4710(3)$ & $34(1)$ \\
\hline$C(25)$ & 1865(6) & 1579(3) & $-6408(3)$ & $48(2)$ \\
\hline$C(26)$ & $4860(6)$ & 2191(3) & $-6770(3)$ & $52(2)$ \\
\hline$C(27)$ & $6544(5)$ & $3762(3)$ & $-5314(3)$ & $42(1)$ \\
\hline $\mathrm{C}(28)$ & $4598(5)$ & $4159(2)$ & $-4048(2)$ & $34(1)$ \\
\hline $\mathrm{C}(29)$ & $5448(5)$ & 2211(2) & $-4185(2)$ & $22(1)$ \\
\hline$C(30)$ & $6740(5)$ & $2382(2)$ & $-4443(2)$ & $21(1)$ \\
\hline$C(31)$ & $6631(5)$ & $1776(2)$ & $-5204(2)$ & $21(1)$ \\
\hline$C(32)$ & $5270(5)$ & $1230(2)$ & $-5400(2)$ & $22(1)$ \\
\hline$C(33)$ & $4554(5)$ & $1497(2)$ & $-4778(2)$ & $25(1)$ \\
\hline $\mathrm{C}(34)$ & $5106(5)$ & 2679(3) & $-3419(2)$ & $33(1)$ \\
\hline $\mathrm{C}(35)$ & $8039(5)$ & $3048(3)$ & $-3996(2)$ & $32(1)$ \\
\hline$C(36)$ & $7770(5)$ & 1696(3) & $-5686(2)$ & $31(1)$ \\
\hline $\mathrm{C}(37)$ & $4761(5)$ & $484(2)$ & $-6119(3)$ & $37(1)$ \\
\hline $\mathrm{C}(38)$ & $3132(5)$ & 1089(3) & $-4733(3)$ & $36(1)$ \\
\hline C(39) & $-4457(5)$ & $1219(2)$ & $485(2)$ & $24(1)$ \\
\hline $\mathrm{C}(40)$ & $-4357(5)$ & $862(2)$ & $-298(2)$ & $23(1)$ \\
\hline $\mathrm{C}(41)$ & $-3289(5)$ & $333(2)$ & $-404(2)$ & $20(1)$ \\
\hline $\mathrm{C}(42)$ & $-2733(4)$ & $363(2)$ & $309(2)$ & $18(1)$ \\
\hline$C(43)$ & $-3442(5)$ & $914(2)$ & $858(2)$ & $22(1)$ \\
\hline$C(44)$ & $-5372(5)$ & 1834(2) & $867(3)$ & $33(1)$ \\
\hline $\mathrm{C}(45)$ & $-5154(5)$ & 1029(3) & $-895(2)$ & $31(1)$ \\
\hline$C(46)$ & $-2797(5)$ & $-144(2)$ & $-1131(2)$ & $27(1)$ \\
\hline
\end{tabular}




\begin{tabular}{|c|c|c|c|c|}
\hline $\mathrm{C}(47)$ & $-1579(5)$ & $-80(3)$ & $467(2)$ & $28(1)$ \\
\hline$C(48)$ & $-3160(5)$ & 1161(3) & $1688(2)$ & $33(1)$ \\
\hline C(49) & $3390(5)$ & $5589(2)$ & $-210(2)$ & $20(1)$ \\
\hline$C(50)$ & $2889(5)$ & $4747(2)$ & $-636(2)$ & $20(1)$ \\
\hline $\mathrm{C}(51)$ & $3925(5)$ & 4432(2) & $-1111(2)$ & $18(1)$ \\
\hline$C(52)$ & $2574(5)$ & $6156(3)$ & $328(2)$ & $32(1)$ \\
\hline $\mathrm{C}(53)$ & $1510(5)$ & $4304(2)$ & $-605(2)$ & $28(1)$ \\
\hline$C(54)$ & $3834(5)$ & $3580(2)$ & $-1675(2)$ & $28(1)$ \\
\hline$C(55)$ & 4931(4) & 4923(2) & $976(2)$ & $18(1)$ \\
\hline$C(56)$ & $5287(5)$ & 4209(2) & $417(2)$ & $22(1)$ \\
\hline$C(57)$ & $3687(5)$ & 4993(3) & $1393(2)$ & $29(1)$ \\
\hline $\mathrm{C}(58)$ & $4432(5)$ & $3383(2)$ & $117(2)$ & $29(1)$ \\
\hline $\mathrm{O}(1)$ & $3301(4)$ & $4289(2)$ & $4127(2)$ & $45(1)$ \\
\hline $\mathrm{O}(2)$ & $-990(5)$ & $5321(2)$ & $4443(2)$ & $55(1)$ \\
\hline $\mathrm{O}(3)$ & $-656(4)$ & $2780(2)$ & $3618(2)$ & $48(1)$ \\
\hline $\mathrm{O}(4)$ & $4306(4)$ & $3564(2)$ & $2353(2)$ & $55(1)$ \\
\hline $\mathrm{O}(5)$ & $838(4)$ & 1701(2) & $1714(2)$ & $51(1)$ \\
\hline $\mathrm{O}(6)$ & $788(4)$ & $-217(2)$ & $-2323(2)$ & $42(1)$ \\
\hline $\mathrm{O}(7)$ & $4688(4)$ & $1583(2)$ & $-2398(2)$ & $52(1)$ \\
\hline $\mathrm{O}(8)$ & $883(4)$ & $-66(2)$ & $-4322(2)$ & $42(1)$ \\
\hline $\mathrm{O}(9)$ & $1235(3)$ & $2232(2)$ & $-3329(2)$ & $28(1)$ \\
\hline $\mathrm{O}(10)$ & $-2524(4)$ & $922(2)$ & $-4199(2)$ & $33(1)$ \\
\hline $\mathrm{O}(11)$ & $-1807(3)$ & $3242(2)$ & $-2092(2)$ & $33(1)$ \\
\hline $\mathrm{O}(12)$ & $-2797(4)$ & $624(2)$ & $-2249(2)$ & $40(1)$ \\
\hline$S(1)$ & $1288(1)$ & $4713(1)$ & $2702(1)$ & $26(1)$ \\
\hline$S(2)$ & $-1371(1)$ & $3345(1)$ & $2174(1)$ & $26(1)$ \\
\hline$S(3)$ & $970(1)$ & $2028(1)$ & $-1632(1)$ & $20(1)$ \\
\hline$S(4)$ & $787(1)$ & $3190(1)$ & $839(1)$ & $35(1)$ \\
\hline $\mathrm{Fe}(1)$ & 281(1) & 4078(1) & $3299(1)$ & $22(1)$ \\
\hline $\mathrm{Fe}(2)$ & $1058(1)$ & $3362(1)$ & 2049(1) & $26(1)$ \\
\hline $\mathrm{Fe}(3)$ & $1453(1)$ & 1079(1) & $-2713(1)$ & $22(1)$ \\
\hline $\mathrm{Fe}(4)$ & $-904(1)$ & 1679(1) & $-2631(1)$ & $19(1)$ \\
\hline $\operatorname{Co}(1)$ & $4833(1)$ & 2353(1) & $-5127(1)$ & $16(1)$ \\
\hline $\operatorname{Co}(2)$ & -5000 & 0 & 0 & $15(1)$ \\
\hline $\operatorname{Co}(3)$ & 5000 & 5000 & 0 & $13(1)$ \\
\hline
\end{tabular}


Table 3. Bond lengths $[\AA]$ and angles $\left[{ }^{\circ}\right]$ for $\mathbf{2}$.

\begin{tabular}{|c|c|}
\hline $\mathrm{C}(1)-\mathrm{O}(1)$ & $1.142(5)$ \\
\hline $\mathrm{C}(1)-\mathrm{Fe}(1)$ & $1.778(5)$ \\
\hline $\mathrm{C}(2)-\mathrm{O}(2)$ & $1.138(5)$ \\
\hline $\mathrm{C}(2)-\mathrm{Fe}(1)$ & $1.798(5)$ \\
\hline $\mathrm{C}(3)-\mathrm{O}(3)$ & $1.154(5)$ \\
\hline $\mathrm{C}(3)-\mathrm{Fe}(1)$ & $1.755(5)$ \\
\hline $\mathrm{C}(4)-\mathrm{C}(5)$ & $1.513(7)$ \\
\hline $\mathrm{C}(4)-\mathrm{S}(1)$ & $1.820(4)$ \\
\hline $\mathrm{C}(4)-\mathrm{H}(4 \mathrm{~A})$ & 0.9900 \\
\hline $\mathrm{C}(4)-\mathrm{H}(4 \mathrm{~B})$ & 0.9900 \\
\hline $\mathrm{C}(5)-\mathrm{C}(6)$ & $1.506(6)$ \\
\hline $\mathrm{C}(5)-\mathrm{H}(5 \mathrm{~A})$ & 0.9900 \\
\hline $\mathrm{C}(5)-\mathrm{H}(5 \mathrm{~B})$ & 0.9900 \\
\hline$C(6)-S(2)$ & $1.829(4)$ \\
\hline $\mathrm{C}(6)-\mathrm{H}(6 \mathrm{~A})$ & 0.9900 \\
\hline $\mathrm{C}(6)-\mathrm{H}(6 \mathrm{~B})$ & 0.9900 \\
\hline $\mathrm{C}(7)-\mathrm{O}(4)$ & $1.157(5)$ \\
\hline $\mathrm{C}(7)-\mathrm{Fe}(2)$ & $1.752(6)$ \\
\hline $\mathrm{C}(8)-\mathrm{O}(5)$ & $1.163(5)$ \\
\hline $\mathrm{C}(8)-\mathrm{Fe}(2)$ & $1.744(5)$ \\
\hline $\mathrm{C}(9)-\mathrm{C}(10)$ & $1.513(6)$ \\
\hline $\mathrm{C}(9)-\mathrm{S}(4)$ & $1.817(4)$ \\
\hline $\mathrm{C}(9)-\mathrm{H}(9 \mathrm{~A})$ & 0.9900 \\
\hline $\mathrm{C}(9)-\mathrm{H}(9 \mathrm{~B})$ & 0.9900 \\
\hline$C(10)-C(11)$ & $1.518(6)$ \\
\hline $\mathrm{C}(10)-\mathrm{H}(10 \mathrm{~A})$ & 0.9900 \\
\hline $\mathrm{C}(10)-\mathrm{H}(10 \mathrm{~B})$ & 0.9900 \\
\hline$C(11)-S(3)$ & $1.829(4)$ \\
\hline $\mathrm{C}(11)-\mathrm{H}(11 \mathrm{~A})$ & 0.9900 \\
\hline $\mathrm{C}(11)-\mathrm{H}(11 \mathrm{~B})$ & 0.9900 \\
\hline $\mathrm{C}(12)-\mathrm{O}(6)$ & $1.148(5)$ \\
\hline $\mathrm{C}(12)-\mathrm{Fe}(3)$ & $1.800(5)$ \\
\hline $\mathrm{C}(13)-\mathrm{O}(7)$ & $1.146(5)$ \\
\hline $\mathrm{C}(13)-\mathrm{Fe}(3)$ & $1.777(5)$ \\
\hline
\end{tabular}




\begin{tabular}{|c|c|}
\hline $\mathrm{C}(14)-\mathrm{O}(8)$ & $1.145(5)$ \\
\hline $\mathrm{C}(14)-\mathrm{Fe}(3)$ & $1.774(5)$ \\
\hline $\mathrm{C}(15)-\mathrm{O}(9)$ & $1.186(4)$ \\
\hline $\mathrm{C}(15)-\mathrm{Fe}(4)$ & $1.940(4)$ \\
\hline $\mathrm{C}(15)-\mathrm{Fe}(3)$ & $1.964(4)$ \\
\hline $\mathrm{C}(16)-\mathrm{O}(10)$ & $1.142(5)$ \\
\hline $\mathrm{C}(16)-\mathrm{Fe}(4)$ & $1.771(5)$ \\
\hline $\mathrm{C}(17)-\mathrm{O}(11)$ & $1.141(5)$ \\
\hline C(17)-Fe(4) & $1.776(5)$ \\
\hline $\mathrm{C}(18)-\mathrm{O}(12)$ & $1.141(5)$ \\
\hline $\mathrm{C}(18)-\mathrm{Fe}(4)$ & $1.823(4)$ \\
\hline$C(19)-C(20)$ & $1.433(6)$ \\
\hline C(19)-C(23) & $1.440(5)$ \\
\hline C(19)-C(24) & $1.490(6)$ \\
\hline C(19)-Co(1) & $2.051(4)$ \\
\hline $\mathrm{C}(20)-\mathrm{C}(21)$ & $1.420(6)$ \\
\hline$C(20)-C(25)$ & $1.505(6)$ \\
\hline $\mathrm{C}(20)-\mathrm{Co}(1)$ & $2.038(4)$ \\
\hline $\mathrm{C}(21)-\mathrm{C}(22)$ & $1.419(6)$ \\
\hline$C(21)-C(26)$ & $1.492(6)$ \\
\hline $\mathrm{C}(21)-\operatorname{Co}(1)$ & $2.041(4)$ \\
\hline$C(22)-C(23)$ & $1.408(6)$ \\
\hline $\mathrm{C}(22)-\mathrm{C}(27)$ & $1.500(6)$ \\
\hline $\mathrm{C}(22)-\mathrm{Co}(1)$ & $2.039(4)$ \\
\hline $\mathrm{C}(23)-\mathrm{C}(28)$ & $1.487(5)$ \\
\hline $\mathrm{C}(23)-\mathrm{Co}(1)$ & $2.048(4)$ \\
\hline $\mathrm{C}(24)-\mathrm{H}(24 \mathrm{~A})$ & 0.9800 \\
\hline $\mathrm{C}(24)-\mathrm{H}(24 \mathrm{~B})$ & 0.9800 \\
\hline $\mathrm{C}(24)-\mathrm{H}(24 \mathrm{C})$ & 0.9800 \\
\hline $\mathrm{C}(25)-\mathrm{H}(25 \mathrm{~A})$ & 0.9800 \\
\hline $\mathrm{C}(25)-\mathrm{H}(25 \mathrm{~B})$ & 0.9800 \\
\hline $\mathrm{C}(25)-\mathrm{H}(25 \mathrm{C})$ & 0.9800 \\
\hline $\mathrm{C}(26)-\mathrm{H}(26 \mathrm{~A})$ & 0.9800 \\
\hline $\mathrm{C}(26)-\mathrm{H}(26 \mathrm{~B})$ & 0.9800 \\
\hline $\mathrm{C}(26)-\mathrm{H}(26 \mathrm{C})$ & 0.9800 \\
\hline $\mathrm{C}(27)-\mathrm{H}(27 \mathrm{~A})$ & 0.9800 \\
\hline
\end{tabular}




\begin{tabular}{|c|c|}
\hline $\mathrm{C}(27)-\mathrm{H}(27 \mathrm{~B})$ & 0.9800 \\
\hline $\mathrm{C}(27)-\mathrm{H}(27 \mathrm{C})$ & 0.9800 \\
\hline $\mathrm{C}(28)-\mathrm{H}(28 \mathrm{~A})$ & 0.9800 \\
\hline $\mathrm{C}(28)-\mathrm{H}(28 \mathrm{~B})$ & 0.9800 \\
\hline $\mathrm{C}(28)-\mathrm{H}(28 \mathrm{C})$ & 0.9800 \\
\hline C(29)-C(33) & $1.422(5)$ \\
\hline C(29)-C(30) & $1.425(6)$ \\
\hline C(29)-C(34) & $1.491(6)$ \\
\hline $\mathrm{C}(29)-\mathrm{Co}(1)$ & $2.043(4)$ \\
\hline C(30)-C(31) & $1.430(5)$ \\
\hline $\mathrm{C}(30)-\mathrm{C}(35)$ & $1.500(5)$ \\
\hline $\mathrm{C}(30)-\operatorname{Co}(1)$ & $2.049(4)$ \\
\hline $\mathrm{C}(31)-\mathrm{C}(32)$ & $1.435(5)$ \\
\hline $\mathrm{C}(31)-\mathrm{C}(36)$ & $1.493(6)$ \\
\hline $\mathrm{C}(31)-\mathrm{Co}(1)$ & $2.045(4)$ \\
\hline $\mathrm{C}(32)-\mathrm{C}(33)$ & $1.404(6)$ \\
\hline $\mathrm{C}(32)-\mathrm{C}(37)$ & $1.479(5)$ \\
\hline $\mathrm{C}(32)-\operatorname{Co}(1)$ & $2.049(4)$ \\
\hline $\mathrm{C}(33)-\mathrm{C}(38)$ & $1.493(6)$ \\
\hline $\mathrm{C}(33)-\operatorname{Co}(1)$ & $2.053(4)$ \\
\hline $\mathrm{C}(34)-\mathrm{H}(34 \mathrm{~A})$ & 0.9800 \\
\hline $\mathrm{C}(34)-\mathrm{H}(34 \mathrm{~B})$ & 0.9800 \\
\hline $\mathrm{C}(34)-\mathrm{H}(34 \mathrm{C})$ & 0.9800 \\
\hline $\mathrm{C}(35)-\mathrm{H}(35 \mathrm{~A})$ & 0.9800 \\
\hline $\mathrm{C}(35)-\mathrm{H}(35 \mathrm{~B})$ & 0.9800 \\
\hline $\mathrm{C}(35)-\mathrm{H}(35 \mathrm{C})$ & 0.9800 \\
\hline $\mathrm{C}(36)-\mathrm{H}(36 \mathrm{~A})$ & 0.9800 \\
\hline $\mathrm{C}(36)-\mathrm{H}(36 \mathrm{~B})$ & 0.9800 \\
\hline $\mathrm{C}(36)-\mathrm{H}(36 \mathrm{C})$ & 0.9800 \\
\hline C(37)-H(37A) & 0.9800 \\
\hline $\mathrm{C}(37)-\mathrm{H}(37 \mathrm{~B})$ & 0.9800 \\
\hline $\mathrm{C}(37)-\mathrm{H}(37 \mathrm{C})$ & 0.9800 \\
\hline $\mathrm{C}(38)-\mathrm{H}(38 \mathrm{~A})$ & 0.9800 \\
\hline $\mathrm{C}(38)-\mathrm{H}(38 \mathrm{~B})$ & 0.9800 \\
\hline C(38)-H(38C) & 0.9800 \\
\hline $\mathrm{C}(39)-\mathrm{C}(40)$ & $1.430(6)$ \\
\hline
\end{tabular}




\begin{tabular}{|c|c|}
\hline C(39)-C(43) & $1.434(6)$ \\
\hline C(39)-C(44) & $1.486(6)$ \\
\hline C(39)-Co(2) & $2.055(4)$ \\
\hline $\mathrm{C}(40)-\mathrm{C}(41)$ & $1.433(6)$ \\
\hline C(40)-C(45) & $1.491(6)$ \\
\hline C(40)-Co(2) & $2.051(4)$ \\
\hline $\mathrm{C}(41)-\mathrm{C}(42)$ & $1.429(5)$ \\
\hline $\mathrm{C}(41)-\mathrm{C}(46)$ & $1.494(5)$ \\
\hline $\mathrm{C}(41)-\mathrm{Co}(2)$ & $2.041(4)$ \\
\hline $\mathrm{C}(42)-\mathrm{C}(43)$ & $1.422(6)$ \\
\hline $\mathrm{C}(42)-\mathrm{C}(47)$ & $1.495(5)$ \\
\hline $\mathrm{C}(42)-\mathrm{Co}(2)$ & $2.045(4)$ \\
\hline C(43)-C(48) & $1.495(5)$ \\
\hline C(43)-Co(2) & $2.058(4)$ \\
\hline C(44)-H(44A) & 0.9800 \\
\hline $\mathrm{C}(44)-\mathrm{H}(44 \mathrm{~B})$ & 0.9800 \\
\hline C(44)-H(44C) & 0.9800 \\
\hline $\mathrm{C}(45)-\mathrm{H}(45 \mathrm{~A})$ & 0.9800 \\
\hline C(45)-H(45B) & 0.9800 \\
\hline $\mathrm{C}(45)-\mathrm{H}(45 \mathrm{C})$ & 0.9800 \\
\hline $\mathrm{C}(46)-\mathrm{H}(46 \mathrm{~A})$ & 0.9800 \\
\hline $\mathrm{C}(46)-\mathrm{H}(46 \mathrm{~B})$ & 0.9800 \\
\hline C(46)-H(46C) & 0.9800 \\
\hline C(47)-H(47A) & 0.9800 \\
\hline $\mathrm{C}(47)-\mathrm{H}(47 \mathrm{~B})$ & 0.9800 \\
\hline C(47)-H(47C) & 0.9800 \\
\hline $\mathrm{C}(48)-\mathrm{H}(48 \mathrm{~A})$ & 0.9800 \\
\hline $\mathrm{C}(48)-\mathrm{H}(48 \mathrm{~B})$ & 0.9800 \\
\hline $\mathrm{C}(48)-\mathrm{H}(48 \mathrm{C})$ & 0.9800 \\
\hline $\mathrm{C}(49)-\mathrm{C}(56) \# 1$ & $1.412(6)$ \\
\hline C(49)-C(50) & $1.431(5)$ \\
\hline C(49)-C(52) & $1.489(6)$ \\
\hline $\mathrm{C}(49)-\operatorname{Co}(3)$ & $2.039(4)$ \\
\hline $\mathrm{C}(50)-\mathrm{C}(51)$ & $1.419(6)$ \\
\hline $\mathrm{C}(50)-\mathrm{C}(53)$ & $1.482(5)$ \\
\hline $\mathrm{C}(50)-\mathrm{Co}(3)$ & $2.051(4)$ \\
\hline
\end{tabular}




\begin{tabular}{|c|c|}
\hline$C(51)-C(55) \# 1$ & $1.441(5)$ \\
\hline$C(51)-C(54)$ & $1.492(5)$ \\
\hline $\mathrm{C}(51)-\mathrm{Co}(3)$ & $2.043(4)$ \\
\hline $\mathrm{C}(52)-\mathrm{H}(52 \mathrm{~A})$ & 0.9800 \\
\hline $\mathrm{C}(52)-\mathrm{H}(52 \mathrm{~B})$ & 0.9800 \\
\hline $\mathrm{C}(52)-\mathrm{H}(52 \mathrm{C})$ & 0.9800 \\
\hline $\mathrm{C}(53)-\mathrm{H}(53 \mathrm{~A})$ & 0.9800 \\
\hline $\mathrm{C}(53)-\mathrm{H}(53 \mathrm{~B})$ & 0.9800 \\
\hline $\mathrm{C}(53)-\mathrm{H}(53 \mathrm{C})$ & 0.9800 \\
\hline $\mathrm{C}(54)-\mathrm{H}(54 \mathrm{~A})$ & 0.9800 \\
\hline $\mathrm{C}(54)-\mathrm{H}(54 \mathrm{~B})$ & 0.9800 \\
\hline $\mathrm{C}(54)-\mathrm{H}(54 \mathrm{C})$ & 0.9800 \\
\hline$C(55)-C(56)$ & $1.426(5)$ \\
\hline $\mathrm{C}(55)-\mathrm{C}(51) \# 1$ & $1.441(5)$ \\
\hline $\mathrm{C}(55)-\mathrm{C}(57)$ & $1.488(6)$ \\
\hline $\mathrm{C}(55)-\mathrm{Co}(3)$ & $2.043(4)$ \\
\hline $\mathrm{C}(56)-\mathrm{C}(49) \# 1$ & $1.412(6)$ \\
\hline $\mathrm{C}(56)-\mathrm{C}(58)$ & $1.500(5)$ \\
\hline $\mathrm{C}(56)-\mathrm{Co}(3)$ & $2.047(4)$ \\
\hline $\mathrm{C}(57)-\mathrm{H}(57 \mathrm{~A})$ & 0.9800 \\
\hline $\mathrm{C}(57)-\mathrm{H}(57 \mathrm{~B})$ & 0.9800 \\
\hline $\mathrm{C}(57)-\mathrm{H}(57 \mathrm{C})$ & 0.9800 \\
\hline $\mathrm{C}(58)-\mathrm{H}(58 \mathrm{~A})$ & 0.9800 \\
\hline $\mathrm{C}(58)-\mathrm{H}(58 \mathrm{~B})$ & 0.9800 \\
\hline $\mathrm{C}(58)-\mathrm{H}(58 \mathrm{C})$ & 0.9800 \\
\hline $\mathrm{S}(1)-\mathrm{Fe}(1)$ & $2.2733(15)$ \\
\hline $\mathrm{S}(1)-\mathrm{Fe}(2)$ & $2.2774(19)$ \\
\hline $\mathrm{S}(2)-\mathrm{Fe}(2)$ & $2.273(2)$ \\
\hline $\mathrm{S}(2)-\mathrm{Fe}(1)$ & $2.2818(17)$ \\
\hline $\mathrm{S}(3)-\mathrm{Fe}(4)$ & $2.2534(17)$ \\
\hline $\mathrm{S}(3)-\mathrm{Fe}(3)$ & $2.2545(16)$ \\
\hline $\mathrm{S}(4)-\mathrm{Fe}(2)$ & $2.280(2)$ \\
\hline $\mathrm{Fe}(1)-\mathrm{Fe}(2)$ & $2.5075(16)$ \\
\hline $\mathrm{Fe}(3)-\mathrm{Fe}(4)$ & $2.5224(16)$ \\
\hline $\mathrm{Co}(2)-\mathrm{C}(41) \# 2$ & $2.041(4)$ \\
\hline $\mathrm{Co}(2)-\mathrm{C}(42) \# 2$ & $2.045(4)$ \\
\hline
\end{tabular}




\begin{tabular}{|c|c|}
\hline $\mathrm{Co}(2)-\mathrm{C}(40) \# 2$ & $2.051(4)$ \\
\hline $\mathrm{Co}(2)-\mathrm{C}(39) \# 2$ & $2.055(4)$ \\
\hline $\mathrm{Co}(2)-\mathrm{C}(43) \# 2$ & $2.058(4)$ \\
\hline Co(3)-C(49)\#1 & $2.039(4)$ \\
\hline $\mathrm{Co}(3)-\mathrm{C}(51) \# 1$ & $2.043(4)$ \\
\hline $\mathrm{Co}(3)-\mathrm{C}(55) \# 1$ & $2.043(4)$ \\
\hline $\mathrm{Co}(3)-\mathrm{C}(56) \# 1$ & $2.047(4)$ \\
\hline $\mathrm{Co}(3)-\mathrm{C}(50) \# 1$ & $2.051(4)$ \\
\hline $\mathrm{O}(1)-\mathrm{C}(1)-\mathrm{Fe}(1)$ & 178.1(4) \\
\hline $\mathrm{O}(2)-\mathrm{C}(2)-\mathrm{Fe}(1)$ & $176.0(4)$ \\
\hline $\mathrm{O}(3)-\mathrm{C}(3)-\mathrm{Fe}(1)$ & $179.3(5)$ \\
\hline$C(5)-C(4)-S(1)$ & $115.9(3)$ \\
\hline $\mathrm{C}(5)-\mathrm{C}(4)-\mathrm{H}(4 \mathrm{~A})$ & 108.3 \\
\hline $\mathrm{S}(1)-\mathrm{C}(4)-\mathrm{H}(4 \mathrm{~A})$ & 108.3 \\
\hline $\mathrm{C}(5)-\mathrm{C}(4)-\mathrm{H}(4 \mathrm{~B})$ & 108.3 \\
\hline $\mathrm{S}(1)-\mathrm{C}(4)-\mathrm{H}(4 \mathrm{~B})$ & 108.3 \\
\hline $\mathrm{H}(4 \mathrm{~A})-\mathrm{C}(4)-\mathrm{H}(4 \mathrm{~B})$ & 107.4 \\
\hline$C(6)-C(5)-C(4)$ & $112.9(4)$ \\
\hline $\mathrm{C}(6)-\mathrm{C}(5)-\mathrm{H}(5 \mathrm{~A})$ & 109.0 \\
\hline $\mathrm{C}(4)-\mathrm{C}(5)-\mathrm{H}(5 \mathrm{~A})$ & 109.0 \\
\hline $\mathrm{C}(6)-\mathrm{C}(5)-\mathrm{H}(5 \mathrm{~B})$ & 109.0 \\
\hline $\mathrm{C}(4)-\mathrm{C}(5)-\mathrm{H}(5 \mathrm{~B})$ & 109.0 \\
\hline $\mathrm{H}(5 \mathrm{~A})-\mathrm{C}(5)-\mathrm{H}(5 \mathrm{~B})$ & 107.8 \\
\hline$C(5)-C(6)-S(2)$ & 116.2(3) \\
\hline $\mathrm{C}(5)-\mathrm{C}(6)-\mathrm{H}(6 \mathrm{~A})$ & 108.2 \\
\hline $\mathrm{S}(2)-\mathrm{C}(6)-\mathrm{H}(6 \mathrm{~A})$ & 108.2 \\
\hline $\mathrm{C}(5)-\mathrm{C}(6)-\mathrm{H}(6 \mathrm{~B})$ & 108.2 \\
\hline $\mathrm{S}(2)-\mathrm{C}(6)-\mathrm{H}(6 \mathrm{~B})$ & 108.2 \\
\hline $\mathrm{H}(6 \mathrm{~A})-\mathrm{C}(6)-\mathrm{H}(6 \mathrm{~B})$ & 107.4 \\
\hline $\mathrm{O}(4)-\mathrm{C}(7)-\mathrm{Fe}(2)$ & $178.0(4)$ \\
\hline $\mathrm{O}(5)-\mathrm{C}(8)-\mathrm{Fe}(2)$ & $177.6(5)$ \\
\hline $\mathrm{C}(10)-\mathrm{C}(9)-\mathrm{S}(4)$ & 109.2(3) \\
\hline $\mathrm{C}(10)-\mathrm{C}(9)-\mathrm{H}(9 \mathrm{~A})$ & 109.8 \\
\hline $\mathrm{S}(4)-\mathrm{C}(9)-\mathrm{H}(9 \mathrm{~A})$ & 109.8 \\
\hline $\mathrm{C}(10)-\mathrm{C}(9)-\mathrm{H}(9 \mathrm{~B})$ & 109.8 \\
\hline
\end{tabular}




\begin{tabular}{|c|c|}
\hline $\mathrm{S}(4)-\mathrm{C}(9)-\mathrm{H}(9 \mathrm{~B})$ & 109.8 \\
\hline $\mathrm{H}(9 \mathrm{~A})-\mathrm{C}(9)-\mathrm{H}(9 \mathrm{~B})$ & 108.3 \\
\hline $\mathrm{C}(9)-\mathrm{C}(10)-\mathrm{C}(11)$ & $114.5(4)$ \\
\hline $\mathrm{C}(9)-\mathrm{C}(10)-\mathrm{H}(10 \mathrm{~A})$ & 108.6 \\
\hline $\mathrm{C}(11)-\mathrm{C}(10)-\mathrm{H}(10 \mathrm{~A})$ & 108.6 \\
\hline $\mathrm{C}(9)-\mathrm{C}(10)-\mathrm{H}(10 \mathrm{~B})$ & 108.6 \\
\hline $\mathrm{C}(11)-\mathrm{C}(10)-\mathrm{H}(10 \mathrm{~B})$ & 108.6 \\
\hline $\mathrm{H}(10 \mathrm{~A})-\mathrm{C}(10)-\mathrm{H}(10 \mathrm{~B})$ & 107.6 \\
\hline$C(10)-C(11)-S(3)$ & 111.7(3) \\
\hline $\mathrm{C}(10)-\mathrm{C}(11)-\mathrm{H}(11 \mathrm{~A})$ & 109.3 \\
\hline $\mathrm{S}(3)-\mathrm{C}(11)-\mathrm{H}(11 \mathrm{~A})$ & 109.3 \\
\hline $\mathrm{C}(10)-\mathrm{C}(11)-\mathrm{H}(11 \mathrm{~B})$ & 109.3 \\
\hline $\mathrm{S}(3)-\mathrm{C}(11)-\mathrm{H}(11 \mathrm{~B})$ & 109.3 \\
\hline $\mathrm{H}(11 \mathrm{~A})-\mathrm{C}(11)-\mathrm{H}(11 \mathrm{~B})$ & 108.0 \\
\hline $\mathrm{O}(6)-\mathrm{C}(12)-\mathrm{Fe}(3)$ & $177.8(4)$ \\
\hline $\mathrm{O}(7)-\mathrm{C}(13)-\mathrm{Fe}(3)$ & 179.0(4) \\
\hline $\mathrm{O}(8)-\mathrm{C}(14)-\mathrm{Fe}(3)$ & $177.6(4)$ \\
\hline $\mathrm{O}(9)-\mathrm{C}(15)-\mathrm{Fe}(4)$ & 139.1(3) \\
\hline $\mathrm{O}(9)-\mathrm{C}(15)-\mathrm{Fe}(3)$ & $140.2(3)$ \\
\hline $\mathrm{Fe}(4)-\mathrm{C}(15)-\mathrm{Fe}(3)$ & $80.50(16)$ \\
\hline $\mathrm{O}(10)-\mathrm{C}(16)-\mathrm{Fe}(4)$ & $178.2(4)$ \\
\hline $\mathrm{O}(11)-\mathrm{C}(17)-\mathrm{Fe}(4)$ & $177.7(4)$ \\
\hline $\mathrm{O}(12)-\mathrm{C}(18)-\mathrm{Fe}(4)$ & $177.4(4)$ \\
\hline $\mathrm{C}(20)-\mathrm{C}(19)-\mathrm{C}(23)$ & $107.1(4)$ \\
\hline $\mathrm{C}(20)-\mathrm{C}(19)-\mathrm{C}(24)$ & $126.8(4)$ \\
\hline $\mathrm{C}(23)-\mathrm{C}(19)-\mathrm{C}(24)$ & $126.1(4)$ \\
\hline $\mathrm{C}(20)-\mathrm{C}(19)-\mathrm{Co}(1)$ & $69.0(2)$ \\
\hline $\mathrm{C}(23)-\mathrm{C}(19)-\mathrm{Co}(1)$ & $69.3(2)$ \\
\hline$C(24)-C(19)-\operatorname{Co}(1)$ & $127.8(3)$ \\
\hline$C(21)-C(20)-C(19)$ & $108.0(4)$ \\
\hline$C(21)-C(20)-C(25)$ & $126.4(4)$ \\
\hline$C(19)-C(20)-C(25)$ & $125.5(4)$ \\
\hline $\mathrm{C}(21)-\mathrm{C}(20)-\mathrm{Co}(1)$ & $69.7(3)$ \\
\hline $\mathrm{C}(19)-\mathrm{C}(20)-\mathrm{Co}(1)$ & $70.0(2)$ \\
\hline $\mathrm{C}(25)-\mathrm{C}(20)-\mathrm{Co}(1)$ & $127.5(3)$ \\
\hline$C(22)-C(21)-C(20)$ & $108.2(4)$ \\
\hline
\end{tabular}




\begin{tabular}{|c|c|}
\hline$C(22)-C(21)-C(26)$ & $125.9(5)$ \\
\hline $\mathrm{C}(20)-\mathrm{C}(21)-\mathrm{C}(26)$ & $125.9(5)$ \\
\hline $\mathrm{C}(22)-\mathrm{C}(21)-\mathrm{Co}(1)$ & $69.6(2)$ \\
\hline $\mathrm{C}(20)-\mathrm{C}(21)-\mathrm{Co}(1)$ & $69.5(2)$ \\
\hline $\mathrm{C}(26)-\mathrm{C}(21)-\mathrm{Co}(1)$ & $127.9(3)$ \\
\hline$C(23)-C(22)-C(21)$ & $108.6(4)$ \\
\hline $\mathrm{C}(23)-\mathrm{C}(22)-\mathrm{C}(27)$ & $126.0(4)$ \\
\hline $\mathrm{C}(21)-\mathrm{C}(22)-\mathrm{C}(27)$ & $125.4(4)$ \\
\hline $\mathrm{C}(23)-\mathrm{C}(22)-\mathrm{Co}(1)$ & $70.2(2)$ \\
\hline $\mathrm{C}(21)-\mathrm{C}(22)-\mathrm{Co}(1)$ & $69.7(2)$ \\
\hline $\mathrm{C}(27)-\mathrm{C}(22)-\mathrm{Co}(1)$ & $127.6(3)$ \\
\hline $\mathrm{C}(22)-\mathrm{C}(23)-\mathrm{C}(19)$ & $108.1(4)$ \\
\hline $\mathrm{C}(22)-\mathrm{C}(23)-\mathrm{C}(28)$ & $126.1(4)$ \\
\hline $\mathrm{C}(19)-\mathrm{C}(23)-\mathrm{C}(28)$ & $125.8(4)$ \\
\hline $\mathrm{C}(22)-\mathrm{C}(23)-\mathrm{Co}(1)$ & $69.5(2)$ \\
\hline $\mathrm{C}(19)-\mathrm{C}(23)-\mathrm{Co}(1)$ & $69.5(2)$ \\
\hline $\mathrm{C}(28)-\mathrm{C}(23)-\mathrm{Co}(1)$ & $128.1(3)$ \\
\hline $\mathrm{C}(19)-\mathrm{C}(24)-\mathrm{H}(24 \mathrm{~A})$ & 109.5 \\
\hline $\mathrm{C}(19)-\mathrm{C}(24)-\mathrm{H}(24 \mathrm{~B})$ & 109.5 \\
\hline $\mathrm{H}(24 \mathrm{~A})-\mathrm{C}(24)-\mathrm{H}(24 \mathrm{~B})$ & 109.5 \\
\hline $\mathrm{C}(19)-\mathrm{C}(24)-\mathrm{H}(24 \mathrm{C})$ & 109.5 \\
\hline $\mathrm{H}(24 \mathrm{~A})-\mathrm{C}(24)-\mathrm{H}(24 \mathrm{C})$ & 109.5 \\
\hline $\mathrm{H}(24 \mathrm{~B})-\mathrm{C}(24)-\mathrm{H}(24 \mathrm{C})$ & 109.5 \\
\hline $\mathrm{C}(20)-\mathrm{C}(25)-\mathrm{H}(25 \mathrm{~A})$ & 109.5 \\
\hline $\mathrm{C}(20)-\mathrm{C}(25)-\mathrm{H}(25 \mathrm{~B})$ & 109.5 \\
\hline $\mathrm{H}(25 \mathrm{~A})-\mathrm{C}(25)-\mathrm{H}(25 \mathrm{~B})$ & 109.5 \\
\hline $\mathrm{C}(20)-\mathrm{C}(25)-\mathrm{H}(25 \mathrm{C})$ & 109.5 \\
\hline $\mathrm{H}(25 \mathrm{~A})-\mathrm{C}(25)-\mathrm{H}(25 \mathrm{C})$ & 109.5 \\
\hline $\mathrm{H}(25 \mathrm{~B})-\mathrm{C}(25)-\mathrm{H}(25 \mathrm{C})$ & 109.5 \\
\hline $\mathrm{C}(21)-\mathrm{C}(26)-\mathrm{H}(26 \mathrm{~A})$ & 109.5 \\
\hline $\mathrm{C}(21)-\mathrm{C}(26)-\mathrm{H}(26 \mathrm{~B})$ & 109.5 \\
\hline $\mathrm{H}(26 \mathrm{~A})-\mathrm{C}(26)-\mathrm{H}(26 \mathrm{~B})$ & 109.5 \\
\hline $\mathrm{C}(21)-\mathrm{C}(26)-\mathrm{H}(26 \mathrm{C})$ & 109.5 \\
\hline $\mathrm{H}(26 \mathrm{~A})-\mathrm{C}(26)-\mathrm{H}(26 \mathrm{C})$ & 109.5 \\
\hline $\mathrm{H}(26 \mathrm{~B})-\mathrm{C}(26)-\mathrm{H}(26 \mathrm{C})$ & 109.5 \\
\hline $\mathrm{C}(22)-\mathrm{C}(27)-\mathrm{H}(27 \mathrm{~A})$ & 109.5 \\
\hline
\end{tabular}




\begin{tabular}{|c|c|}
\hline $\mathrm{C}(22)-\mathrm{C}(27)-\mathrm{H}(27 \mathrm{~B})$ & 109.5 \\
\hline $\mathrm{H}(27 \mathrm{~A})-\mathrm{C}(27)-\mathrm{H}(27 \mathrm{~B})$ & 109.5 \\
\hline $\mathrm{C}(22)-\mathrm{C}(27)-\mathrm{H}(27 \mathrm{C})$ & 109.5 \\
\hline $\mathrm{H}(27 \mathrm{~A})-\mathrm{C}(27)-\mathrm{H}(27 \mathrm{C})$ & 109.5 \\
\hline $\mathrm{H}(27 \mathrm{~B})-\mathrm{C}(27)-\mathrm{H}(27 \mathrm{C})$ & 109.5 \\
\hline $\mathrm{C}(23)-\mathrm{C}(28)-\mathrm{H}(28 \mathrm{~A})$ & 109.5 \\
\hline $\mathrm{C}(23)-\mathrm{C}(28)-\mathrm{H}(28 \mathrm{~B})$ & 109.5 \\
\hline $\mathrm{H}(28 \mathrm{~A})-\mathrm{C}(28)-\mathrm{H}(28 \mathrm{~B})$ & 109.5 \\
\hline $\mathrm{C}(23)-\mathrm{C}(28)-\mathrm{H}(28 \mathrm{C})$ & 109.5 \\
\hline $\mathrm{H}(28 \mathrm{~A})-\mathrm{C}(28)-\mathrm{H}(28 \mathrm{C})$ & 109.5 \\
\hline $\mathrm{H}(28 \mathrm{~B})-\mathrm{C}(28)-\mathrm{H}(28 \mathrm{C})$ & 109.5 \\
\hline$C(33)-C(29)-C(30)$ & $108.3(4)$ \\
\hline$C(33)-C(29)-C(34)$ & $125.4(4)$ \\
\hline$C(30)-C(29)-C(34)$ & $126.3(4)$ \\
\hline $\mathrm{C}(33)-\mathrm{C}(29)-\mathrm{Co}(1)$ & $70.1(2)$ \\
\hline $\mathrm{C}(30)-\mathrm{C}(29)-\mathrm{Co}(1)$ & $69.9(2)$ \\
\hline $\mathrm{C}(34)-\mathrm{C}(29)-\mathrm{Co}(1)$ & $127.2(3)$ \\
\hline $\mathrm{C}(29)-\mathrm{C}(30)-\mathrm{C}(31)$ & $107.8(4)$ \\
\hline$C(29)-C(30)-C(35)$ & $127.2(4)$ \\
\hline$C(31)-C(30)-C(35)$ & $125.0(4)$ \\
\hline $\mathrm{C}(29)-\mathrm{C}(30)-\mathrm{Co}(1)$ & $69.4(2)$ \\
\hline $\mathrm{C}(31)-\mathrm{C}(30)-\mathrm{Co}(1)$ & $69.4(2)$ \\
\hline $\mathrm{C}(35)-\mathrm{C}(30)-\mathrm{Co}(1)$ & 129.1(3) \\
\hline $\mathrm{C}(30)-\mathrm{C}(31)-\mathrm{C}(32)$ & 107.1(4) \\
\hline$C(30)-C(31)-C(36)$ & $126.3(4)$ \\
\hline $\mathrm{C}(32)-\mathrm{C}(31)-\mathrm{C}(36)$ & $126.4(4)$ \\
\hline $\mathrm{C}(30)-\mathrm{C}(31)-\mathrm{Co}(1)$ & $69.7(2)$ \\
\hline $\mathrm{C}(32)-\mathrm{C}(31)-\mathrm{Co}(1)$ & $69.6(2)$ \\
\hline $\mathrm{C}(36)-\mathrm{C}(31)-\mathrm{Co}(1)$ & $128.9(3)$ \\
\hline $\mathrm{C}(33)-\mathrm{C}(32)-\mathrm{C}(31)$ & $108.7(4)$ \\
\hline $\mathrm{C}(33)-\mathrm{C}(32)-\mathrm{C}(37)$ & $125.8(4)$ \\
\hline $\mathrm{C}(31)-\mathrm{C}(32)-\mathrm{C}(37)$ & $125.4(4)$ \\
\hline $\mathrm{C}(33)-\mathrm{C}(32)-\mathrm{Co}(1)$ & $70.2(2)$ \\
\hline $\mathrm{C}(31)-\mathrm{C}(32)-\mathrm{Co}(1)$ & $69.3(2)$ \\
\hline $\mathrm{C}(37)-\mathrm{C}(32)-\mathrm{Co}(1)$ & $129.3(3)$ \\
\hline $\mathrm{C}(32)-\mathrm{C}(33)-\mathrm{C}(29)$ & $108.1(4)$ \\
\hline
\end{tabular}




\begin{tabular}{|c|c|}
\hline $\mathrm{C}(32)-\mathrm{C}(33)-\mathrm{C}(38)$ & $126.1(4)$ \\
\hline $\mathrm{C}(29)-\mathrm{C}(33)-\mathrm{C}(38)$ & $125.7(4)$ \\
\hline $\mathrm{C}(32)-\mathrm{C}(33)-\mathrm{Co}(1)$ & $69.8(2)$ \\
\hline $\mathrm{C}(29)-\mathrm{C}(33)-\mathrm{Co}(1)$ & $69.3(2)$ \\
\hline $\mathrm{C}(38)-\mathrm{C}(33)-\mathrm{Co}(1)$ & $128.4(3)$ \\
\hline $\mathrm{C}(29)-\mathrm{C}(34)-\mathrm{H}(34 \mathrm{~A})$ & 109.5 \\
\hline $\mathrm{C}(29)-\mathrm{C}(34)-\mathrm{H}(34 \mathrm{~B})$ & 109.5 \\
\hline $\mathrm{H}(34 \mathrm{~A})-\mathrm{C}(34)-\mathrm{H}(34 \mathrm{~B})$ & 109.5 \\
\hline $\mathrm{C}(29)-\mathrm{C}(34)-\mathrm{H}(34 \mathrm{C})$ & 109.5 \\
\hline $\mathrm{H}(34 \mathrm{~A})-\mathrm{C}(34)-\mathrm{H}(34 \mathrm{C})$ & 109.5 \\
\hline $\mathrm{H}(34 \mathrm{~B})-\mathrm{C}(34)-\mathrm{H}(34 \mathrm{C})$ & 109.5 \\
\hline $\mathrm{C}(30)-\mathrm{C}(35)-\mathrm{H}(35 \mathrm{~A})$ & 109.5 \\
\hline $\mathrm{C}(30)-\mathrm{C}(35)-\mathrm{H}(35 \mathrm{~B})$ & 109.5 \\
\hline $\mathrm{H}(35 \mathrm{~A})-\mathrm{C}(35)-\mathrm{H}(35 \mathrm{~B})$ & 109.5 \\
\hline $\mathrm{C}(30)-\mathrm{C}(35)-\mathrm{H}(35 \mathrm{C})$ & 109.5 \\
\hline $\mathrm{H}(35 \mathrm{~A})-\mathrm{C}(35)-\mathrm{H}(35 \mathrm{C})$ & 109.5 \\
\hline $\mathrm{H}(35 \mathrm{~B})-\mathrm{C}(35)-\mathrm{H}(35 \mathrm{C})$ & 109.5 \\
\hline $\mathrm{C}(31)-\mathrm{C}(36)-\mathrm{H}(36 \mathrm{~A})$ & 109.5 \\
\hline $\mathrm{C}(31)-\mathrm{C}(36)-\mathrm{H}(36 \mathrm{~B})$ & 109.5 \\
\hline $\mathrm{H}(36 \mathrm{~A})-\mathrm{C}(36)-\mathrm{H}(36 \mathrm{~B})$ & 109.5 \\
\hline $\mathrm{C}(31)-\mathrm{C}(36)-\mathrm{H}(36 \mathrm{C})$ & 109.5 \\
\hline $\mathrm{H}(36 \mathrm{~A})-\mathrm{C}(36)-\mathrm{H}(36 \mathrm{C})$ & 109.5 \\
\hline $\mathrm{H}(36 \mathrm{~B})-\mathrm{C}(36)-\mathrm{H}(36 \mathrm{C})$ & 109.5 \\
\hline $\mathrm{C}(32)-\mathrm{C}(37)-\mathrm{H}(37 \mathrm{~A})$ & 109.5 \\
\hline $\mathrm{C}(32)-\mathrm{C}(37)-\mathrm{H}(37 \mathrm{~B})$ & 109.5 \\
\hline $\mathrm{H}(37 \mathrm{~A})-\mathrm{C}(37)-\mathrm{H}(37 \mathrm{~B})$ & 109.5 \\
\hline $\mathrm{C}(32)-\mathrm{C}(37)-\mathrm{H}(37 \mathrm{C})$ & 109.5 \\
\hline $\mathrm{H}(37 \mathrm{~A})-\mathrm{C}(37)-\mathrm{H}(37 \mathrm{C})$ & 109.5 \\
\hline $\mathrm{H}(37 \mathrm{~B})-\mathrm{C}(37)-\mathrm{H}(37 \mathrm{C})$ & 109.5 \\
\hline $\mathrm{C}(33)-\mathrm{C}(38)-\mathrm{H}(38 \mathrm{~A})$ & 109.5 \\
\hline $\mathrm{C}(33)-\mathrm{C}(38)-\mathrm{H}(38 \mathrm{~B})$ & 109.5 \\
\hline $\mathrm{H}(38 \mathrm{~A})-\mathrm{C}(38)-\mathrm{H}(38 \mathrm{~B})$ & 109.5 \\
\hline $\mathrm{C}(33)-\mathrm{C}(38)-\mathrm{H}(38 \mathrm{C})$ & 109.5 \\
\hline $\mathrm{H}(38 \mathrm{~A})-\mathrm{C}(38)-\mathrm{H}(38 \mathrm{C})$ & 109.5 \\
\hline $\mathrm{H}(38 \mathrm{~B})-\mathrm{C}(38)-\mathrm{H}(38 \mathrm{C})$ & 109.5 \\
\hline $\mathrm{C}(40)-\mathrm{C}(39)-\mathrm{C}(43)$ & $107.8(4)$ \\
\hline
\end{tabular}




\begin{tabular}{|c|c|}
\hline $\mathrm{C}(40)-\mathrm{C}(39)-\mathrm{C}(44)$ & $126.9(4)$ \\
\hline $\mathrm{C}(43)-\mathrm{C}(39)-\mathrm{C}(44)$ & $125.2(4)$ \\
\hline $\mathrm{C}(40)-\mathrm{C}(39)-\mathrm{Co}(2)$ & $69.5(2)$ \\
\hline $\mathrm{C}(43)-\mathrm{C}(39)-\mathrm{Co}(2)$ & $69.7(2)$ \\
\hline $\mathrm{C}(44)-\mathrm{C}(39)-\mathrm{Co}(2)$ & $129.0(3)$ \\
\hline $\mathrm{C}(39)-\mathrm{C}(40)-\mathrm{C}(41)$ & $107.6(4)$ \\
\hline$C(39)-C(40)-C(45)$ & $126.8(4)$ \\
\hline $\mathrm{C}(41)-\mathrm{C}(40)-\mathrm{C}(45)$ & $125.5(4)$ \\
\hline $\mathrm{C}(39)-\mathrm{C}(40)-\mathrm{Co}(2)$ & $69.8(2)$ \\
\hline $\mathrm{C}(41)-\mathrm{C}(40)-\mathrm{Co}(2)$ & $69.1(2)$ \\
\hline $\mathrm{C}(45)-\mathrm{C}(40)-\mathrm{Co}(2)$ & $128.6(3)$ \\
\hline$C(42)-C(41)-C(40)$ & $108.4(4)$ \\
\hline$C(42)-C(41)-C(46)$ & $125.8(4)$ \\
\hline $\mathrm{C}(40)-\mathrm{C}(41)-\mathrm{C}(46)$ & $125.8(4)$ \\
\hline $\mathrm{C}(42)-\mathrm{C}(41)-\mathrm{Co}(2)$ & $69.7(2)$ \\
\hline $\mathrm{C}(40)-\mathrm{C}(41)-\mathrm{Co}(2)$ & $69.9(2)$ \\
\hline $\mathrm{C}(46)-\mathrm{C}(41)-\mathrm{Co}(2)$ & $127.9(3)$ \\
\hline $\mathrm{C}(43)-\mathrm{C}(42)-\mathrm{C}(41)$ & $107.8(4)$ \\
\hline$C(43)-C(42)-C(47)$ & $125.4(4)$ \\
\hline $\mathrm{C}(41)-\mathrm{C}(42)-\mathrm{C}(47)$ & $126.8(4)$ \\
\hline $\mathrm{C}(43)-\mathrm{C}(42)-\mathrm{Co}(2)$ & $70.2(2)$ \\
\hline $\mathrm{C}(41)-\mathrm{C}(42)-\mathrm{Co}(2)$ & $69.4(2)$ \\
\hline $\mathrm{C}(47)-\mathrm{C}(42)-\mathrm{Co}(2)$ & $127.3(3)$ \\
\hline$C(42)-C(43)-C(39)$ & $108.4(4)$ \\
\hline $\mathrm{C}(42)-\mathrm{C}(43)-\mathrm{C}(48)$ & $126.6(4)$ \\
\hline $\mathrm{C}(39)-\mathrm{C}(43)-\mathrm{C}(48)$ & $124.9(4)$ \\
\hline $\mathrm{C}(42)-\mathrm{C}(43)-\mathrm{Co}(2)$ & $69.3(2)$ \\
\hline $\mathrm{C}(39)-\mathrm{C}(43)-\mathrm{Co}(2)$ & $69.5(2)$ \\
\hline $\mathrm{C}(48)-\mathrm{C}(43)-\mathrm{Co}(2)$ & $128.5(3)$ \\
\hline $\mathrm{C}(39)-\mathrm{C}(44)-\mathrm{H}(44 \mathrm{~A})$ & 109.5 \\
\hline $\mathrm{C}(39)-\mathrm{C}(44)-\mathrm{H}(44 \mathrm{~B})$ & 109.5 \\
\hline $\mathrm{H}(44 \mathrm{~A})-\mathrm{C}(44)-\mathrm{H}(44 \mathrm{~B})$ & 109.5 \\
\hline C(39)-C(44)-H(44C) & 109.5 \\
\hline $\mathrm{H}(44 \mathrm{~A})-\mathrm{C}(44)-\mathrm{H}(44 \mathrm{C})$ & 109.5 \\
\hline $\mathrm{H}(44 \mathrm{~B})-\mathrm{C}(44)-\mathrm{H}(44 \mathrm{C})$ & 109.5 \\
\hline C(40)-C(45)-H(45A) & 109.5 \\
\hline
\end{tabular}




\begin{tabular}{|c|c|}
\hline $\mathrm{C}(40)-\mathrm{C}(45)-\mathrm{H}(45 \mathrm{~B})$ & 109.5 \\
\hline $\mathrm{H}(45 \mathrm{~A})-\mathrm{C}(45)-\mathrm{H}(45 \mathrm{~B})$ & 109.5 \\
\hline $\mathrm{C}(40)-\mathrm{C}(45)-\mathrm{H}(45 \mathrm{C})$ & 109.5 \\
\hline $\mathrm{H}(45 \mathrm{~A})-\mathrm{C}(45)-\mathrm{H}(45 \mathrm{C})$ & 109.5 \\
\hline $\mathrm{H}(45 \mathrm{~B})-\mathrm{C}(45)-\mathrm{H}(45 \mathrm{C})$ & 109.5 \\
\hline $\mathrm{C}(41)-\mathrm{C}(46)-\mathrm{H}(46 \mathrm{~A})$ & 109.5 \\
\hline $\mathrm{C}(41)-\mathrm{C}(46)-\mathrm{H}(46 \mathrm{~B})$ & 109.5 \\
\hline $\mathrm{H}(46 \mathrm{~A})-\mathrm{C}(46)-\mathrm{H}(46 \mathrm{~B})$ & 109.5 \\
\hline $\mathrm{C}(41)-\mathrm{C}(46)-\mathrm{H}(46 \mathrm{C})$ & 109.5 \\
\hline $\mathrm{H}(46 \mathrm{~A})-\mathrm{C}(46)-\mathrm{H}(46 \mathrm{C})$ & 109.5 \\
\hline $\mathrm{H}(46 \mathrm{~B})-\mathrm{C}(46)-\mathrm{H}(46 \mathrm{C})$ & 109.5 \\
\hline $\mathrm{C}(42)-\mathrm{C}(47)-\mathrm{H}(47 \mathrm{~A})$ & 109.5 \\
\hline $\mathrm{C}(42)-\mathrm{C}(47)-\mathrm{H}(47 \mathrm{~B})$ & 109.5 \\
\hline $\mathrm{H}(47 \mathrm{~A})-\mathrm{C}(47)-\mathrm{H}(47 \mathrm{~B})$ & 109.5 \\
\hline C(42)-C(47)-H(47C) & 109.5 \\
\hline $\mathrm{H}(47 \mathrm{~A})-\mathrm{C}(47)-\mathrm{H}(47 \mathrm{C})$ & 109.5 \\
\hline $\mathrm{H}(47 \mathrm{~B})-\mathrm{C}(47)-\mathrm{H}(47 \mathrm{C})$ & 109.5 \\
\hline $\mathrm{C}(43)-\mathrm{C}(48)-\mathrm{H}(48 \mathrm{~A})$ & 109.5 \\
\hline $\mathrm{C}(43)-\mathrm{C}(48)-\mathrm{H}(48 \mathrm{~B})$ & 109.5 \\
\hline $\mathrm{H}(48 \mathrm{~A})-\mathrm{C}(48)-\mathrm{H}(48 \mathrm{~B})$ & 109.5 \\
\hline C(43)-C(48)-H(48C) & 109.5 \\
\hline $\mathrm{H}(48 \mathrm{~A})-\mathrm{C}(48)-\mathrm{H}(48 \mathrm{C})$ & 109.5 \\
\hline $\mathrm{H}(48 \mathrm{~B})-\mathrm{C}(48)-\mathrm{H}(48 \mathrm{C})$ & 109.5 \\
\hline $\mathrm{C}(56) \# 1-\mathrm{C}(49)-\mathrm{C}(50)$ & 109.2(4) \\
\hline C(56)\#1-C(49)-C(52) & $126.2(4)$ \\
\hline $\mathrm{C}(50)-\mathrm{C}(49)-\mathrm{C}(52)$ & $124.4(4)$ \\
\hline C(56)\#1-C(49)-Co(3) & $70.1(2)$ \\
\hline $\mathrm{C}(50)-\mathrm{C}(49)-\mathrm{Co}(3)$ & $70.0(2)$ \\
\hline $\mathrm{C}(52)-\mathrm{C}(49)-\mathrm{Co}(3)$ & $128.9(3)$ \\
\hline$C(51)-C(50)-C(49)$ & $106.9(4)$ \\
\hline$C(51)-C(50)-C(53)$ & $127.2(4)$ \\
\hline$C(49)-C(50)-C(53)$ & $125.9(4)$ \\
\hline $\mathrm{C}(51)-\mathrm{C}(50)-\mathrm{Co}(3)$ & $69.4(2)$ \\
\hline $\mathrm{C}(49)-\mathrm{C}(50)-\mathrm{Co}(3)$ & $69.0(2)$ \\
\hline $\mathrm{C}(53)-\mathrm{C}(50)-\mathrm{Co}(3)$ & $128.6(3)$ \\
\hline $\mathrm{C}(50)-\mathrm{C}(51)-\mathrm{C}(55) \# 1$ & $108.7(3)$ \\
\hline
\end{tabular}




\begin{tabular}{|c|c|}
\hline$C(50)-C(51)-C(54)$ & $126.1(4)$ \\
\hline $\mathrm{C}(55) \# 1-\mathrm{C}(51)-\mathrm{C}(54)$ & $125.2(4)$ \\
\hline $\mathrm{C}(50)-\mathrm{C}(51)-\mathrm{Co}(3)$ & $70.0(2)$ \\
\hline $\mathrm{C}(55) \# 1-\mathrm{C}(51)-\mathrm{Co}(3)$ & $69.4(2)$ \\
\hline $\mathrm{C}(54)-\mathrm{C}(51)-\mathrm{Co}(3)$ & $127.9(3)$ \\
\hline $\mathrm{C}(49)-\mathrm{C}(52)-\mathrm{H}(52 \mathrm{~A})$ & 109.5 \\
\hline $\mathrm{C}(49)-\mathrm{C}(52)-\mathrm{H}(52 \mathrm{~B})$ & 109.5 \\
\hline $\mathrm{H}(52 \mathrm{~A})-\mathrm{C}(52)-\mathrm{H}(52 \mathrm{~B})$ & 109.5 \\
\hline $\mathrm{C}(49)-\mathrm{C}(52)-\mathrm{H}(52 \mathrm{C})$ & 109.5 \\
\hline $\mathrm{H}(52 \mathrm{~A})-\mathrm{C}(52)-\mathrm{H}(52 \mathrm{C})$ & 109.5 \\
\hline $\mathrm{H}(52 \mathrm{~B})-\mathrm{C}(52)-\mathrm{H}(52 \mathrm{C})$ & 109.5 \\
\hline $\mathrm{C}(50)-\mathrm{C}(53)-\mathrm{H}(53 \mathrm{~A})$ & 109.5 \\
\hline $\mathrm{C}(50)-\mathrm{C}(53)-\mathrm{H}(53 \mathrm{~B})$ & 109.5 \\
\hline $\mathrm{H}(53 \mathrm{~A})-\mathrm{C}(53)-\mathrm{H}(53 \mathrm{~B})$ & 109.5 \\
\hline $\mathrm{C}(50)-\mathrm{C}(53)-\mathrm{H}(53 \mathrm{C})$ & 109.5 \\
\hline $\mathrm{H}(53 \mathrm{~A})-\mathrm{C}(53)-\mathrm{H}(53 \mathrm{C})$ & 109.5 \\
\hline $\mathrm{H}(53 \mathrm{~B})-\mathrm{C}(53)-\mathrm{H}(53 \mathrm{C})$ & 109.5 \\
\hline $\mathrm{C}(51)-\mathrm{C}(54)-\mathrm{H}(54 \mathrm{~A})$ & 109.5 \\
\hline $\mathrm{C}(51)-\mathrm{C}(54)-\mathrm{H}(54 \mathrm{~B})$ & 109.5 \\
\hline $\mathrm{H}(54 \mathrm{~A})-\mathrm{C}(54)-\mathrm{H}(54 \mathrm{~B})$ & 109.5 \\
\hline $\mathrm{C}(51)-\mathrm{C}(54)-\mathrm{H}(54 \mathrm{C})$ & 109.5 \\
\hline $\mathrm{H}(54 \mathrm{~A})-\mathrm{C}(54)-\mathrm{H}(54 \mathrm{C})$ & 109.5 \\
\hline $\mathrm{H}(54 \mathrm{~B})-\mathrm{C}(54)-\mathrm{H}(54 \mathrm{C})$ & 109.5 \\
\hline $\mathrm{C}(56)-\mathrm{C}(55)-\mathrm{C}(51) \# 1$ & 107.3(4) \\
\hline $\mathrm{C}(56)-\mathrm{C}(55)-\mathrm{C}(57)$ & $126.9(4)$ \\
\hline $\mathrm{C}(51) \# 1-\mathrm{C}(55)-\mathrm{C}(57)$ & $125.7(4)$ \\
\hline $\mathrm{C}(56)-\mathrm{C}(55)-\mathrm{Co}(3)$ & $69.7(2)$ \\
\hline $\mathrm{C}(51) \# 1-\mathrm{C}(55)-\mathrm{Co}(3)$ & $69.3(2)$ \\
\hline$C(57)-C(55)-C o(3)$ & $129.8(3)$ \\
\hline C(49)\#1-C(56)-C(55) & $108.0(3)$ \\
\hline C(49)\#1-C(56)-C(58) & $125.9(4)$ \\
\hline $\mathrm{C}(55)-\mathrm{C}(56)-\mathrm{C}(58)$ & 126.1(4) \\
\hline C(49)\#1-C(56)-Co(3) & $69.5(2)$ \\
\hline $\mathrm{C}(55)-\mathrm{C}(56)-\mathrm{Co}(3)$ & $69.5(2)$ \\
\hline $\mathrm{C}(58)-\mathrm{C}(56)-\mathrm{Co}(3)$ & $127.9(3)$ \\
\hline $\mathrm{C}(55)-\mathrm{C}(57)-\mathrm{H}(57 \mathrm{~A})$ & 109.5 \\
\hline
\end{tabular}




\begin{tabular}{|c|c|}
\hline $\mathrm{C}(55)-\mathrm{C}(57)-\mathrm{H}(57 \mathrm{~B})$ & 109.5 \\
\hline $\mathrm{H}(57 \mathrm{~A})-\mathrm{C}(57)-\mathrm{H}(57 \mathrm{~B})$ & 109.5 \\
\hline C(55)-C(57)-H(57C) & 109.5 \\
\hline $\mathrm{H}(57 \mathrm{~A})-\mathrm{C}(57)-\mathrm{H}(57 \mathrm{C})$ & 109.5 \\
\hline $\mathrm{H}(57 \mathrm{~B})-\mathrm{C}(57)-\mathrm{H}(57 \mathrm{C})$ & 109.5 \\
\hline $\mathrm{C}(56)-\mathrm{C}(58)-\mathrm{H}(58 \mathrm{~A})$ & 109.5 \\
\hline $\mathrm{C}(56)-\mathrm{C}(58)-\mathrm{H}(58 \mathrm{~B})$ & 109.5 \\
\hline $\mathrm{H}(58 \mathrm{~A})-\mathrm{C}(58)-\mathrm{H}(58 \mathrm{~B})$ & 109.5 \\
\hline $\mathrm{C}(56)-\mathrm{C}(58)-\mathrm{H}(58 \mathrm{C})$ & 109.5 \\
\hline $\mathrm{H}(58 \mathrm{~A})-\mathrm{C}(58)-\mathrm{H}(58 \mathrm{C})$ & 109.5 \\
\hline $\mathrm{H}(58 \mathrm{~B})-\mathrm{C}(58)-\mathrm{H}(58 \mathrm{C})$ & 109.5 \\
\hline $\mathrm{C}(4)-\mathrm{S}(1)-\mathrm{Fe}(1)$ & $112.67(17)$ \\
\hline $\mathrm{C}(4)-\mathrm{S}(1)-\mathrm{Fe}(2)$ & $111.45(15)$ \\
\hline $\mathrm{Fe}(1)-\mathrm{S}(1)-\mathrm{Fe}(2)$ & $66.87(6)$ \\
\hline $\mathrm{C}(6)-\mathrm{S}(2)-\mathrm{Fe}(2)$ & $111.80(16)$ \\
\hline $\mathrm{C}(6)-\mathrm{S}(2)-\mathrm{Fe}(1)$ & $112.02(15)$ \\
\hline $\mathrm{Fe}(2)-\mathrm{S}(2)-\mathrm{Fe}(1)$ & $66.80(5)$ \\
\hline $\mathrm{C}(11)-\mathrm{S}(3)-\mathrm{Fe}(4)$ & $113.64(15)$ \\
\hline $\mathrm{C}(11)-\mathrm{S}(3)-\mathrm{Fe}(3)$ & $117.54(14)$ \\
\hline $\mathrm{Fe}(4)-\mathrm{S}(3)-\mathrm{Fe}(3)$ & $68.05(6)$ \\
\hline C(9)-S(4)-Fe(2) & $107.34(14)$ \\
\hline $\mathrm{C}(3)-\mathrm{Fe}(1)-\mathrm{C}(1)$ & $90.4(2)$ \\
\hline $\mathrm{C}(3)-\mathrm{Fe}(1)-\mathrm{C}(2)$ & $98.8(2)$ \\
\hline $\mathrm{C}(1)-\mathrm{Fe}(1)-\mathrm{C}(2)$ & $102.8(2)$ \\
\hline $\mathrm{C}(3)-\mathrm{Fe}(1)-\mathrm{S}(1)$ & $158.87(15)$ \\
\hline $\mathrm{C}(1)-\mathrm{Fe}(1)-\mathrm{S}(1)$ & $87.05(14)$ \\
\hline $\mathrm{C}(2)-\mathrm{Fe}(1)-\mathrm{S}(1)$ & $102.23(15)$ \\
\hline $\mathrm{C}(3)-\mathrm{Fe}(1)-\mathrm{S}(2)$ & $84.93(16)$ \\
\hline $\mathrm{C}(1)-\mathrm{Fe}(1)-\mathrm{S}(2)$ & $145.39(15)$ \\
\hline $\mathrm{C}(2)-\mathrm{Fe}(1)-\mathrm{S}(2)$ & $111.80(16)$ \\
\hline $\mathrm{S}(1)-\mathrm{Fe}(1)-\mathrm{S}(2)$ & $85.35(7)$ \\
\hline C(3)-Fe(1)-Fe(2) & $102.52(16)$ \\
\hline $\mathrm{C}(1)-\mathrm{Fe}(1)-\mathrm{Fe}(2)$ & $91.57(15)$ \\
\hline $\mathrm{C}(2)-\mathrm{Fe}(1)-\mathrm{Fe}(2)$ & $154.16(13)$ \\
\hline $\mathrm{S}(1)-\mathrm{Fe}(1)-\mathrm{Fe}(2)$ & $56.64(5)$ \\
\hline $\mathrm{S}(2)-\mathrm{Fe}(1)-\mathrm{Fe}(2)$ & $56.44(6)$ \\
\hline
\end{tabular}




\begin{tabular}{|c|c|}
\hline $\mathrm{C}(8)-\mathrm{Fe}(2)-\mathrm{C}(7)$ & $91.3(2)$ \\
\hline $\mathrm{C}(8)-\mathrm{Fe}(2)-\mathrm{S}(2)$ & $90.48(16)$ \\
\hline $\mathrm{C}(7)-\mathrm{Fe}(2)-\mathrm{S}(2)$ & $164.51(16)$ \\
\hline $\mathrm{C}(8)-\mathrm{Fe}(2)-\mathrm{S}(1)$ & $162.02(16)$ \\
\hline $\mathrm{C}(7)-\mathrm{Fe}(2)-\mathrm{S}(1)$ & $88.13(15)$ \\
\hline $\mathrm{S}(2)-\mathrm{Fe}(2)-\mathrm{S}(1)$ & $85.45(5)$ \\
\hline $\mathrm{C}(8)-\mathrm{Fe}(2)-\mathrm{S}(4)$ & $97.75(15)$ \\
\hline $\mathrm{C}(7)-\mathrm{Fe}(2)-\mathrm{S}(4)$ & $95.51(16)$ \\
\hline $\mathrm{S}(2)-\mathrm{Fe}(2)-\mathrm{S}(4)$ & $99.49(5)$ \\
\hline $\mathrm{S}(1)-\mathrm{Fe}(2)-\mathrm{S}(4)$ & $100.20(5)$ \\
\hline $\mathrm{C}(8)-\mathrm{Fe}(2)-\mathrm{Fe}(1)$ & $106.94(15)$ \\
\hline $\mathrm{C}(7)-\mathrm{Fe}(2)-\mathrm{Fe}(1)$ & $108.11(16)$ \\
\hline $\mathrm{S}(2)-\mathrm{Fe}(2)-\mathrm{Fe}(1)$ & $56.76(4)$ \\
\hline $\mathrm{S}(1)-\mathrm{Fe}(2)-\mathrm{Fe}(1)$ & $56.49(3)$ \\
\hline $\mathrm{S}(4)-\mathrm{Fe}(2)-\mathrm{Fe}(1)$ & $144.97(5)$ \\
\hline $\mathrm{C}(14)-\mathrm{Fe}(3)-\mathrm{C}(13)$ & $100.5(2)$ \\
\hline $\mathrm{C}(14)-\mathrm{Fe}(3)-\mathrm{C}(12)$ & 91.91(19) \\
\hline $\mathrm{C}(13)-\mathrm{Fe}(3)-\mathrm{C}(12)$ & $108.5(2)$ \\
\hline $\mathrm{C}(14)-\mathrm{Fe}(3)-\mathrm{C}(15)$ & $87.18(18)$ \\
\hline $\mathrm{C}(13)-\mathrm{Fe}(3)-\mathrm{C}(15)$ & 98.78(19) \\
\hline $\mathrm{C}(12)-\mathrm{Fe}(3)-\mathrm{C}(15)$ & $152.4(2)$ \\
\hline $\mathrm{C}(14)-\mathrm{Fe}(3)-\mathrm{S}(3)$ & $157.35(15)$ \\
\hline $\mathrm{C}(13)-\mathrm{Fe}(3)-\mathrm{S}(3)$ & $98.42(15)$ \\
\hline $\mathrm{C}(12)-\mathrm{Fe}(3)-\mathrm{S}(3)$ & $93.86(14)$ \\
\hline $\mathrm{C}(15)-\mathrm{Fe}(3)-\mathrm{S}(3)$ & $77.65(13)$ \\
\hline $\mathrm{C}(14)-\mathrm{Fe}(3)-\mathrm{Fe}(4)$ & $101.40(15)$ \\
\hline $\mathrm{C}(13)-\mathrm{Fe}(3)-\mathrm{Fe}(4)$ & $139.68(15)$ \\
\hline $\mathrm{C}(12)-\mathrm{Fe}(3)-\mathrm{Fe}(4)$ & $104.16(15)$ \\
\hline $\mathrm{C}(15)-\mathrm{Fe}(3)-\mathrm{Fe}(4)$ & $49.32(13)$ \\
\hline $\mathrm{S}(3)-\mathrm{Fe}(3)-\mathrm{Fe}(4)$ & $55.95(4)$ \\
\hline $\mathrm{C}(16)-\mathrm{Fe}(4)-\mathrm{C}(17)$ & $98.97(18)$ \\
\hline $\mathrm{C}(16)-\mathrm{Fe}(4)-\mathrm{C}(18)$ & $92.71(19)$ \\
\hline $\mathrm{C}(17)-\mathrm{Fe}(4)-\mathrm{C}(18)$ & 109.3(2) \\
\hline $\mathrm{C}(16)-\mathrm{Fe}(4)-\mathrm{C}(15)$ & $86.75(19)$ \\
\hline $\mathrm{C}(17)-\mathrm{Fe}(4)-\mathrm{C}(15)$ & $100.36(18)$ \\
\hline $\mathrm{C}(18)-\mathrm{Fe}(4)-\mathrm{C}(15)$ & $150.06(19)$ \\
\hline
\end{tabular}




\begin{tabular}{|c|c|}
\hline $\mathrm{C}(16)-\mathrm{Fe}(4)-\mathrm{S}(3)$ & $160.09(14)$ \\
\hline $\mathrm{C}(17)-\mathrm{Fe}(4)-\mathrm{S}(3)$ & $96.48(14)$ \\
\hline $\mathrm{C}(18)-\mathrm{Fe}(4)-\mathrm{S}(3)$ & $93.97(14)$ \\
\hline $\mathrm{C}(15)-\mathrm{Fe}(4)-\mathrm{S}(3)$ & $78.16(14)$ \\
\hline $\mathrm{C}(16)-\mathrm{Fe}(4)-\mathrm{Fe}(3)$ & $104.26(14)$ \\
\hline $\mathrm{C}(17)-\mathrm{Fe}(4)-\mathrm{Fe}(3)$ & $140.20(14)$ \\
\hline $\mathrm{C}(18)-\mathrm{Fe}(4)-\mathrm{Fe}(3)$ & $101.49(15)$ \\
\hline $\mathrm{C}(15)-\mathrm{Fe}(4)-\mathrm{Fe}(3)$ & $50.17(13)$ \\
\hline $\mathrm{S}(3)-\mathrm{Fe}(4)-\mathrm{Fe}(3)$ & $56.00(5)$ \\
\hline $\mathrm{C}(20)-\mathrm{Co}(1)-\mathrm{C}(22)$ & $68.65(18)$ \\
\hline $\mathrm{C}(20)-\mathrm{Co}(1)-\mathrm{C}(21)$ & $40.74(18)$ \\
\hline $\mathrm{C}(22)-\mathrm{Co}(1)-\mathrm{C}(21)$ & $40.70(17)$ \\
\hline $\mathrm{C}(20)-\mathrm{Co}(1)-\mathrm{C}(29)$ & $142.90(19)$ \\
\hline $\mathrm{C}(22)-\mathrm{Co}(1)-\mathrm{C}(29)$ & $135.16(17)$ \\
\hline $\mathrm{C}(21)-\mathrm{Co}(1)-\mathrm{C}(29)$ & $175.47(18)$ \\
\hline $\mathrm{C}(20)-\mathrm{Co}(1)-\mathrm{C}(31)$ & $135.53(18)$ \\
\hline $\mathrm{C}(22)-\mathrm{Co}(1)-\mathrm{C}(31)$ & $113.78(17)$ \\
\hline $\mathrm{C}(21)-\mathrm{Co}(1)-\mathrm{C}(31)$ & $110.42(17)$ \\
\hline $\mathrm{C}(29)-\mathrm{Co}(1)-\mathrm{C}(31)$ & $68.68(16)$ \\
\hline $\mathrm{C}(20)-\mathrm{Co}(1)-\mathrm{C}(23)$ & $68.87(17)$ \\
\hline $\mathrm{C}(22)-\mathrm{Co}(1)-\mathrm{C}(23)$ & $40.30(16)$ \\
\hline $\mathrm{C}(21)-\mathrm{Co}(1)-\mathrm{C}(23)$ & $68.31(17)$ \\
\hline $\mathrm{C}(29)-\mathrm{Co}(1)-\mathrm{C}(23)$ & $109.55(16)$ \\
\hline $\mathrm{C}(31)-\mathrm{Co}(1)-\mathrm{C}(23)$ & $143.08(16)$ \\
\hline $\mathrm{C}(20)-\mathrm{Co}(1)-\mathrm{C}(32)$ & $110.11(17)$ \\
\hline $\mathrm{C}(22)-\mathrm{Co}(1)-\mathrm{C}(32)$ & $144.56(17)$ \\
\hline $\mathrm{C}(21)-\mathrm{Co}(1)-\mathrm{C}(32)$ & $114.48(18)$ \\
\hline $\mathrm{C}(29)-\mathrm{Co}(1)-\mathrm{C}(32)$ & $67.99(16)$ \\
\hline $\mathrm{C}(31)-\mathrm{Co}(1)-\mathrm{C}(32)$ & $41.05(15)$ \\
\hline $\mathrm{C}(23)-\mathrm{Co}(1)-\mathrm{C}(32)$ & $174.83(17)$ \\
\hline $\mathrm{C}(20)-\mathrm{Co}(1)-\mathrm{C}(30)$ & $175.80(18)$ \\
\hline $\mathrm{C}(22)-\mathrm{Co}(1)-\mathrm{C}(30)$ & $110.05(17)$ \\
\hline $\mathrm{C}(21)-\mathrm{Co}(1)-\mathrm{C}(30)$ & $135.78(19)$ \\
\hline $\mathrm{C}(29)-\mathrm{Co}(1)-\mathrm{C}(30)$ & $40.75(16)$ \\
\hline $\mathrm{C}(31)-\mathrm{Co}(1)-\mathrm{C}(30)$ & $40.88(16)$ \\
\hline $\mathrm{C}(23)-\mathrm{Co}(1)-\mathrm{C}(30)$ & $112.93(16)$ \\
\hline
\end{tabular}




\begin{tabular}{|c|c|}
\hline $\mathrm{C}(32)-\mathrm{Co}(1)-\mathrm{C}(30)$ & $68.46(16)$ \\
\hline $\mathrm{C}(20)-\mathrm{Co}(1)-\mathrm{C}(19)$ & $41.02(16)$ \\
\hline $\mathrm{C}(22)-\mathrm{Co}(1)-\mathrm{C}(19)$ & $68.62(17)$ \\
\hline $\mathrm{C}(21)-\mathrm{Co}(1)-\mathrm{C}(19)$ & $68.67(18)$ \\
\hline C(29)-Co(1)-C(19) & $112.59(17)$ \\
\hline $\mathrm{C}(31)-\mathrm{Co}(1)-\mathrm{C}(19)$ & $175.51(17)$ \\
\hline $\mathrm{C}(23)-\mathrm{Co}(1)-\mathrm{C}(19)$ & $41.12(15)$ \\
\hline $\mathrm{C}(32)-\mathrm{Co}(1)-\mathrm{C}(19)$ & $134.90(17)$ \\
\hline $\mathrm{C}(30)-\mathrm{Co}(1)-\mathrm{C}(19)$ & $142.73(17)$ \\
\hline $\mathrm{C}(20)-\mathrm{Co}(1)-\mathrm{C}(33)$ & $113.23(18)$ \\
\hline $\mathrm{C}(22)-\mathrm{Co}(1)-\mathrm{C}(33)$ & $174.90(18)$ \\
\hline $\mathrm{C}(21)-\mathrm{Co}(1)-\mathrm{C}(33)$ & $143.64(19)$ \\
\hline $\mathrm{C}(29)-\mathrm{Co}(1)-\mathrm{C}(33)$ & $40.62(15)$ \\
\hline $\mathrm{C}(31)-\mathrm{Co}(1)-\mathrm{C}(33)$ & $68.52(17)$ \\
\hline $\mathrm{C}(23)-\mathrm{Co}(1)-\mathrm{C}(33)$ & $135.24(17)$ \\
\hline $\mathrm{C}(32)-\mathrm{Co}(1)-\mathrm{C}(33)$ & $40.03(16)$ \\
\hline $\mathrm{C}(30)-\mathrm{Co}(1)-\mathrm{C}(33)$ & $68.43(17)$ \\
\hline C(19)-Co(1)-C(33) & $109.43(17)$ \\
\hline $\mathrm{C}(41)-\mathrm{Co}(2)-\mathrm{C}(41) \# 2$ & $180.0(2)$ \\
\hline $\mathrm{C}(41)-\mathrm{Co}(2)-\mathrm{C}(42)$ & $40.93(15)$ \\
\hline $\mathrm{C}(41) \# 2-\mathrm{Co}(2)-\mathrm{C}(42)$ & $139.07(15)$ \\
\hline $\mathrm{C}(41)-\mathrm{Co}(2)-\mathrm{C}(42) \# 2$ & $139.07(15)$ \\
\hline $\mathrm{C}(41) \# 2-\mathrm{Co}(2)-\mathrm{C}(42) \# 2$ & $40.93(15)$ \\
\hline $\mathrm{C}(42)-\mathrm{Co}(2)-\mathrm{C}(42) \# 2$ & 180.0 \\
\hline $\mathrm{C}(41)-\mathrm{Co}(2)-\mathrm{C}(40)$ & $41.00(16)$ \\
\hline $\mathrm{C}(41) \# 2-\mathrm{Co}(2)-\mathrm{C}(40)$ & $139.00(16)$ \\
\hline $\mathrm{C}(42)-\mathrm{Co}(2)-\mathrm{C}(40)$ & $69.01(16)$ \\
\hline $\mathrm{C}(42) \# 2-\mathrm{Co}(2)-\mathrm{C}(40)$ & $110.99(16)$ \\
\hline $\mathrm{C}(41)-\mathrm{Co}(2)-\mathrm{C}(40) \# 2$ & $139.00(16)$ \\
\hline $\mathrm{C}(41) \# 2-\mathrm{Co}(2)-\mathrm{C}(40) \# 2$ & $41.00(16)$ \\
\hline $\mathrm{C}(42)-\mathrm{Co}(2)-\mathrm{C}(40) \# 2$ & $110.99(16)$ \\
\hline $\mathrm{C}(42) \# 2-\mathrm{Co}(2)-\mathrm{C}(40) \# 2$ & $69.01(16)$ \\
\hline $\mathrm{C}(40)-\mathrm{Co}(2)-\mathrm{C}(40) \# 2$ & $180.0(3)$ \\
\hline $\mathrm{C}(41)-\mathrm{Co}(2)-\mathrm{C}(39) \# 2$ & $111.29(16)$ \\
\hline $\mathrm{C}(41) \# 2-\mathrm{Co}(2)-\mathrm{C}(39) \# 2$ & $68.71(16)$ \\
\hline $\mathrm{C}(42)-\mathrm{Co}(2)-\mathrm{C}(39) \# 2$ & $111.19(16)$ \\
\hline
\end{tabular}




\begin{tabular}{|c|c|}
\hline $\mathrm{C}(42) \# 2-\mathrm{Co}(2)-\mathrm{C}(39) \# 2$ & $68.81(16)$ \\
\hline $\mathrm{C}(40)-\mathrm{Co}(2)-\mathrm{C}(39) \# 2$ & 139.23(16) \\
\hline $\mathrm{C}(40) \# 2-\mathrm{Co}(2)-\mathrm{C}(39) \# 2$ & $40.77(16)$ \\
\hline $\mathrm{C}(41)-\mathrm{Co}(2)-\mathrm{C}(39)$ & $68.71(16)$ \\
\hline C(41)\#2-Co(2)-C(39) & $111.29(16)$ \\
\hline $\mathrm{C}(42)-\mathrm{Co}(2)-\mathrm{C}(39)$ & $68.81(16)$ \\
\hline C(42)\#2-Co(2)-C(39) & 111.19(16) \\
\hline $\mathrm{C}(40)-\mathrm{Co}(2)-\mathrm{C}(39)$ & $40.77(16)$ \\
\hline C(40)\#2-Co(2)-C(39) & $139.23(16)$ \\
\hline C(39)\#2-Co(2)-C(39) & $180.0(3)$ \\
\hline $\mathrm{C}(41)-\mathrm{Co}(2)-\mathrm{C}(43)$ & $68.37(16)$ \\
\hline C(41)\#2-Co(2)-C(43) & 111.63(16) \\
\hline $\mathrm{C}(42)-\mathrm{Co}(2)-\mathrm{C}(43)$ & $40.55(16)$ \\
\hline C(42)\#2-Co(2)-C(43) & $139.45(16)$ \\
\hline $\mathrm{C}(40)-\mathrm{Co}(2)-\mathrm{C}(43)$ & $68.56(17)$ \\
\hline $\mathrm{C}(40) \# 2-\mathrm{Co}(2)-\mathrm{C}(43)$ & 111.44(17) \\
\hline C(39)\#2-Co(2)-C(43) & $139.17(16)$ \\
\hline C(39)-Co(2)-C(43) & $40.83(16)$ \\
\hline $\mathrm{C}(41)-\mathrm{Co}(2)-\mathrm{C}(43) \# 2$ & 111.63(16) \\
\hline $\mathrm{C}(41) \# 2-\mathrm{Co}(2)-\mathrm{C}(43) \# 2$ & $68.37(16)$ \\
\hline $\mathrm{C}(42)-\mathrm{Co}(2)-\mathrm{C}(43) \# 2$ & $139.45(16)$ \\
\hline $\mathrm{C}(42) \# 2-\mathrm{Co}(2)-\mathrm{C}(43) \# 2$ & $40.55(16)$ \\
\hline $\mathrm{C}(40)-\mathrm{Co}(2)-\mathrm{C}(43) \# 2$ & $111.44(17)$ \\
\hline $\mathrm{C}(40) \# 2-\mathrm{Co}(2)-\mathrm{C}(43) \# 2$ & $68.56(17)$ \\
\hline $\mathrm{C}(39) \# 2-\mathrm{Co}(2)-\mathrm{C}(43) \# 2$ & $40.83(16)$ \\
\hline $\mathrm{C}(39)-\mathrm{Co}(2)-\mathrm{C}(43) \# 2$ & $139.17(16)$ \\
\hline $\mathrm{C}(43)-\mathrm{Co}(2)-\mathrm{C}(43) \# 2$ & $180.0(4)$ \\
\hline C(49)\#1-Co(3)-C(49) & $180.000(1)$ \\
\hline C(49)\#1-Co(3)-C(51) & $111.76(16)$ \\
\hline C(49)-Co(3)-C(51) & $68.24(16)$ \\
\hline $\mathrm{C}(49) \# 1-\mathrm{Co}(3)-\mathrm{C}(51) \# 1$ & $68.24(16)$ \\
\hline $\mathrm{C}(49)-\mathrm{Co}(3)-\mathrm{C}(51) \# 1$ & $111.76(16)$ \\
\hline $\mathrm{C}(51)-\mathrm{Co}(3)-\mathrm{C}(51) \# 1$ & $180.0(2)$ \\
\hline C(49)\#1-Co(3)-C(55) & $68.44(16)$ \\
\hline $\mathrm{C}(49)-\mathrm{Co}(3)-\mathrm{C}(55)$ & $111.56(16)$ \\
\hline $\mathrm{C}(51)-\mathrm{Co}(3)-\mathrm{C}(55)$ & $138.70(15)$ \\
\hline
\end{tabular}




\begin{tabular}{|c|c|}
\hline C(51)\#1-Co(3)-C(55) & $41.30(15)$ \\
\hline C(49)\#1-Co(3)-C(55)\#1 & $111.56(16)$ \\
\hline $\mathrm{C}(49)-\mathrm{Co}(3)-\mathrm{C}(55) \# 1$ & $68.44(16)$ \\
\hline $\mathrm{C}(51)-\mathrm{Co}(3)-\mathrm{C}(55) \# 1$ & $41.30(15)$ \\
\hline $\mathrm{C}(51) \# 1-\mathrm{Co}(3)-\mathrm{C}(55) \# 1$ & $138.70(15)$ \\
\hline $\mathrm{C}(55)-\mathrm{Co}(3)-\mathrm{C}(55) \# 1$ & $180.0(2)$ \\
\hline C(49)\#1-Co(3)-C(56) & $40.44(16)$ \\
\hline $\mathrm{C}(49)-\mathrm{Co}(3)-\mathrm{C}(56)$ & $139.56(16)$ \\
\hline $\mathrm{C}(51)-\mathrm{Co}(3)-\mathrm{C}(56)$ & $111.27(16)$ \\
\hline C(51)\#1-Co(3)-C(56) & $68.73(16)$ \\
\hline $\mathrm{C}(55)-\mathrm{Co}(3)-\mathrm{C}(56)$ & $40.81(15)$ \\
\hline C(55)\#1-Co(3)-C(56) & $139.19(15)$ \\
\hline $\mathrm{C}(49) \# 1-\mathrm{Co}(3)-\mathrm{C}(56) \# 1$ & $139.56(16)$ \\
\hline C(49)-Co(3)-C(56)\#1 & $40.44(16)$ \\
\hline $\mathrm{C}(51)-\mathrm{Co}(3)-\mathrm{C}(56) \# 1$ & $68.73(16)$ \\
\hline $\mathrm{C}(51) \# 1-\mathrm{Co}(3)-\mathrm{C}(56) \# 1$ & $111.27(16)$ \\
\hline$C(55)-C o(3)-C(56) \# 1$ & $139.19(15)$ \\
\hline $\mathrm{C}(55) \# 1-\mathrm{Co}(3)-\mathrm{C}(56) \# 1$ & $40.81(15)$ \\
\hline $\mathrm{C}(56)-\mathrm{Co}(3)-\mathrm{C}(56) \# 1$ & $180.00(18)$ \\
\hline C(49)\#1-Co(3)-C(50) & $139.04(15)$ \\
\hline C(49)-Co(3)-C(50) & $40.96(15)$ \\
\hline $\mathrm{C}(51)-\mathrm{Co}(3)-\mathrm{C}(50)$ & $40.56(16)$ \\
\hline $\mathrm{C}(51) \# 1-\mathrm{Co}(3)-\mathrm{C}(50)$ & $139.44(16)$ \\
\hline $\mathrm{C}(55)-\mathrm{Co}(3)-\mathrm{C}(50)$ & $110.85(16)$ \\
\hline C(55)\#1-Co(3)-C(50) & $69.15(16)$ \\
\hline $\mathrm{C}(56)-\mathrm{Co}(3)-\mathrm{C}(50)$ & 111.11(16) \\
\hline C(56)\#1-Co(3)-C(50) & $68.89(16)$ \\
\hline C(49)\#1-Co(3)-C(50)\#1 & $40.96(15)$ \\
\hline $\mathrm{C}(49)-\mathrm{Co}(3)-\mathrm{C}(50) \# 1$ & $139.04(15)$ \\
\hline$C(51)-C o(3)-C(50) \# 1$ & $139.44(16)$ \\
\hline $\mathrm{C}(51) \# 1-\mathrm{Co}(3)-\mathrm{C}(50) \# 1$ & $40.56(16)$ \\
\hline $\mathrm{C}(55)-\mathrm{Co}(3)-\mathrm{C}(50) \# 1$ & $69.15(16)$ \\
\hline $\mathrm{C}(55) \# 1-\mathrm{Co}(3)-\mathrm{C}(50) \# 1$ & $110.85(16)$ \\
\hline $\mathrm{C}(56)-\mathrm{Co}(3)-\mathrm{C}(50) \# 1$ & $68.89(16)$ \\
\hline $\mathrm{C}(56) \# 1-\mathrm{Co}(3)-\mathrm{C}(50) \# 1$ & $111.11(16)$ \\
\hline $\mathrm{C}(50)-\mathrm{Co}(3)-\mathrm{C}(50) \# 1$ & $180.0(2)$ \\
\hline
\end{tabular}


Symmetry transformations used to generate equivalent atoms:

$\# 1-x+1,-y+1,-z \quad \# 2-x-1,-y,-z$ 